\title{
THE CONTROL OF FLIGHT FORCE BY A FLAPPING WING: LIFT AND DRAG PRODUCTION
}

\author{
SANJAY P. SANE* AND MICHAEL H. DICKINSON \\ Department of Integrative Biology, University of California, Berkeley, CA 94720, USA \\ *e-mail: sane@socrates.berkeley.edu \\ Accepted 18 May 2001
}

\begin{abstract}
Summary
We used a dynamically scaled mechanical model of the fruit fly Drosophila melanogaster to study how changes in wing kinematics influence the production of unsteady aerodynamic forces in insect flight. We examined 191 separate sets of kinematic patterns that differed with respect to stroke amplitude, angle of attack, flip timing, flip duration and the shape and magnitude of stroke deviation. Instantaneous aerodynamic forces were measured using a two-dimensional force sensor mounted at the base of the wing. The influence of unsteady rotational effects was assessed by comparing the time course of measured forces with that of corresponding translational quasi-steady estimates. For each pattern, we also calculated mean stroke-averaged values of the force coefficients and an estimate of profile power. The results of this analysis may be divided into four main points.

(i) For a short, symmetrical wing flip, mean lift was optimized by a stroke amplitude of $180^{\circ}$ and an angle of attack of $50^{\circ}$. At all stroke amplitudes, mean drag increased monotonically with increasing angle of attack. Translational quasi-steady predictions better matched the measured values at high stroke amplitude than at low stroke amplitude. This discrepancy was due to the increasing importance of rotational mechanisms in kinematic patterns with low stroke amplitude.

(ii) For a $180^{\circ}$ stroke amplitude and a $45^{\circ}$ angle of attack, lift was maximized by short-duration flips occurring just slightly in advance of stroke reversal. Symmetrical rotations produced similarly high

performance. Wing rotation that occurred after stroke reversal, however, produced very low mean lift.

(iii) The production of aerodynamic forces was sensitive to changes in the magnitude of the wing's deviation from the mean stroke plane (stroke deviation) as well as to the actual shape of the wing tip trajectory. However, in all examples, stroke deviation lowered aerodynamic performance relative to the no deviation case. This attenuation was due, in part, to a trade-off between lift and a radially directed component of total aerodynamic force. Thus, while we found no evidence that stroke deviation can augment lift, it nevertheless may be used to modulate forces on the two wings. Thus, insects might use such changes in wing kinematics during steering maneuvers to generate appropriate force moments.

(iv) While quasi-steady estimates failed to capture the time course of measured lift for nearly all kinematic patterns, they did predict with reasonable accuracy stroke-averaged values for the mean lift coefficient. However, quasi-steady estimates grossly underestimated the magnitude of the mean drag coefficient under all conditions. This discrepancy was due to the contribution of rotational effects that steady-state estimates do not capture. This result suggests that many prior estimates of mechanical power based on wing kinematics may have been grossly underestimated.

Key words: flapping flight, quasi-steady force, unsteady aerodynamics, fruit fly, Drosophila melanogaster, added mass, delayed stall, rotational circulation, wake capture.
\end{abstract}

\section{Introduction}

To perform aerial maneuvers, insects must not only generate sufficient lift to remain aloft, they must also manipulate flight forces with great precision. Although insects are known to use their legs and abdomen as control surfaces during flight (Arbas, 1986; Zanker, 1988; May and Hoy, 1990; Lorez, 1995), they steer and maneuver largely by altering wing motion (Götz et al., 1979; Ennos, 1989; Robertson and Johnson, 1993; Wortmann and Zarnack, 1993). Thus, a central hurdle in understanding how insects steer and maneuver is determining how modifications in stroke kinematics alter the forces and moments generated by flapping wings.

In a few cases, researchers have attempted to capture the free-flight kinematics of maneuvering insects (Ennos, 1989; Ruppell, 1989). While such analyses are essential because they reveal what insects actually do with their wings when steering, free-flight studies are limited because it is not yet feasible to relate the changes in wing kinematics directly to changes in instantaneous aerodynamic forces. An alternative approach is 
to measure instantaneous forces on tethered insects (Cloupeau et al., 1979; Wilkin, 1990; Zanker, 1990b; Zanker and Götz, 1990; Dickinson and Götz, 1996). However, forces and stroke kinematics measured on tethered insects may not accurately represent those generated in free flight. Further, since tethered flight force transducers measure whole-body forces, it is not possible to resolve the instantaneous aerodynamic forces generated by individual wings. A third approach is to calculate the aerodynamic forces generated by arbitrary stroke kinematics using computational fluid dynamics (Liu et al., 1998; Wang, 2000). However, because of the critical role of unsteady mechanisms and three-dimensional flow structure in insect flight aerodynamics (Ellington et al., 1996; Dickinson et al., 1999), theoretical or numerical approaches have, as yet, offered only limited insight into the aerodynamics of steering.

Given the current limitations in studies of both real animals and numerical simulations, we have chosen to study the problem of maneuverability using a dynamically scaled model of a flapping insect. Aerodynamic models have proved valuable in the study of insect flight, particularly in the identification and analysis of unsteady aerodynamics (Bennett, 1977; Maxworthy, 1979; Spedding and Maxworthy, 1986; Dickinson and Götz, 1993; Ellington et al., 1996; Dickinson et al., 1999). In large part through the use of mechanical models, researchers have identified an array of mechanisms that collectively account for the elevated aerodynamic performance of flapping wings. These include the clap and fling (Spedding and Maxworthy, 1986), dynamic stall (Dickinson and Götz, 1993; Ellington et al., 1996), rotational lift (Bennett, 1970; Dickinson et al., 1999) and wake capture (Dickinson, 1994; Dickinson et al., 1999). Now that the various mechanisms responsible for the elevated aerodynamic performance of insect wings have been identified, it is possible to tackle the question of how animals manipulate such mechanisms to steer and maneuver.

In this study, we use a dynamically scaled mechanical model of Drosophila melanogaster to investigate how changes in wing kinematics affect the production of aerodynamic forces. In particular, we explore the influence of five behaviorally relevant kinematic parameters: stroke amplitude, angle of attack, the timing and duration of wing rotation and stroke plane deviation. We chose this particular set of parameters because fruit flies actively vary them during flight maneuvers (Götz et al., 1979; Zanker, 1990a; Dickinson et al., 1993; Lehmann and Dickinson, 1998). However, the goal of this project is not to replicate the precise kinematics of free flying insects per se, but rather to map aerodynamic forces within a broad parameter space that encompasses the variation seen among insects.

From the instantaneous force records, we calculate timeaveraged aerodynamic force coefficients, lift-to-drag ratios and other measures of aerodynamic performance. The resultant data set is useful in identifying the kinematic parameters that most influence the magnitude and direction of aerodynamic forces generated by flapping wings. In a companion paper (S. P. Sane and M. H. Dickinson, in preparation), we will extend the analysis by considering the instantaneous and timeaveraged force moments generated about the yaw, pitch and roll axes. The comprehensive parameter maps generated in these studies should be of help to biologists who wish to know the aerodynamic consequences of observed changes in wing kinematics as well as to engineers who wish to optimize the performance of small biomimetic flying robots. In addition, these data provide experimental validations for numerical simulations of the fluid motion around flapping wings.

\section{Materials and methods}

Most of the instruments and procedures used in these experiments have been described elsewhere (Dickinson et al., 1999). We fashioned the wings from $2.3 \mathrm{~mm}$ thick acrylic sheets using an isometrically enlarged planform of a Drosophila melanogaster wing. The proximal end of the wing was attached to a two-dimensional force transducer that measured the forces normal and parallel to the wing surface. Each force channel measured the shear encountered by two parallel phosphor-bronze shims equipped with four $350 \Omega$ strain gauges wired in full-bridge configuration. This design rendered the sensor nearly insensitive to the position of the force load on the wing as well as to moments around its central axis. Forces generated by calibration weights placed at the tip, base, trailing edge and leading edge differed by less than $5 \%$. The final calibration was based on a point load at the wing's center of area. The proximal end of the force transducer was attached to a gearbox capable of three degrees of rotational motion (Fig. 1A). The distal tip of the wing was located $25 \mathrm{~cm}$ from the center of the gearbox. The gearbox was driven via three coaxial shafts by three stepper motors. The stepper motors were attached to the shafts by pulleys and timing belts with a 1:10 gear reduction, such that each $4.5^{\circ}$ step of the motor produced a $0.45^{\circ}$ rotation of the wing. The wings, force sensor and gearbox were immersed in a tank of mineral oil with a viscosity of $120 \mathrm{cSt}$ at room temperature (approximately $25^{\circ} \mathrm{C}$ ). The viscosity of the oil was chosen to achieve a Reynolds number in the range of $10^{2}$, although the exact value varies according to the kinematics for each trial. Since the forces on the wing are directly proportional to the density of the surrounding medium, the oil also serves to increase forces on the wings and to decrease the signal-to-noise ratio of the force measurements. Mineral oil provides an additional advantage of electrically and thermally isolating the sensor and thus reducing noise fluctuations.

\section{Dynamic scaling}

To obtain accurate dynamic scaling of an insect, it is necessary to keep the values of both the Reynolds number ( $R e=4 \Phi n R^{2} \mathrm{v}^{-1} A R^{-1}$, where $\Phi$ is stroke amplitude, $n$ is wingbeat frequency, $R$ is wing length, $v$ is kinematic viscosity, aspect ratio $A R$ is $4 R^{2} S^{-1}$ and $S$ is the surface area of a wing pair; Ellington, $1984 \mathrm{c}$ ) and the reduced frequency parameter (body velocity/wing velocity) constant (Spedding, 1993). For hovering animals as well as the model fly, the reduced frequency 

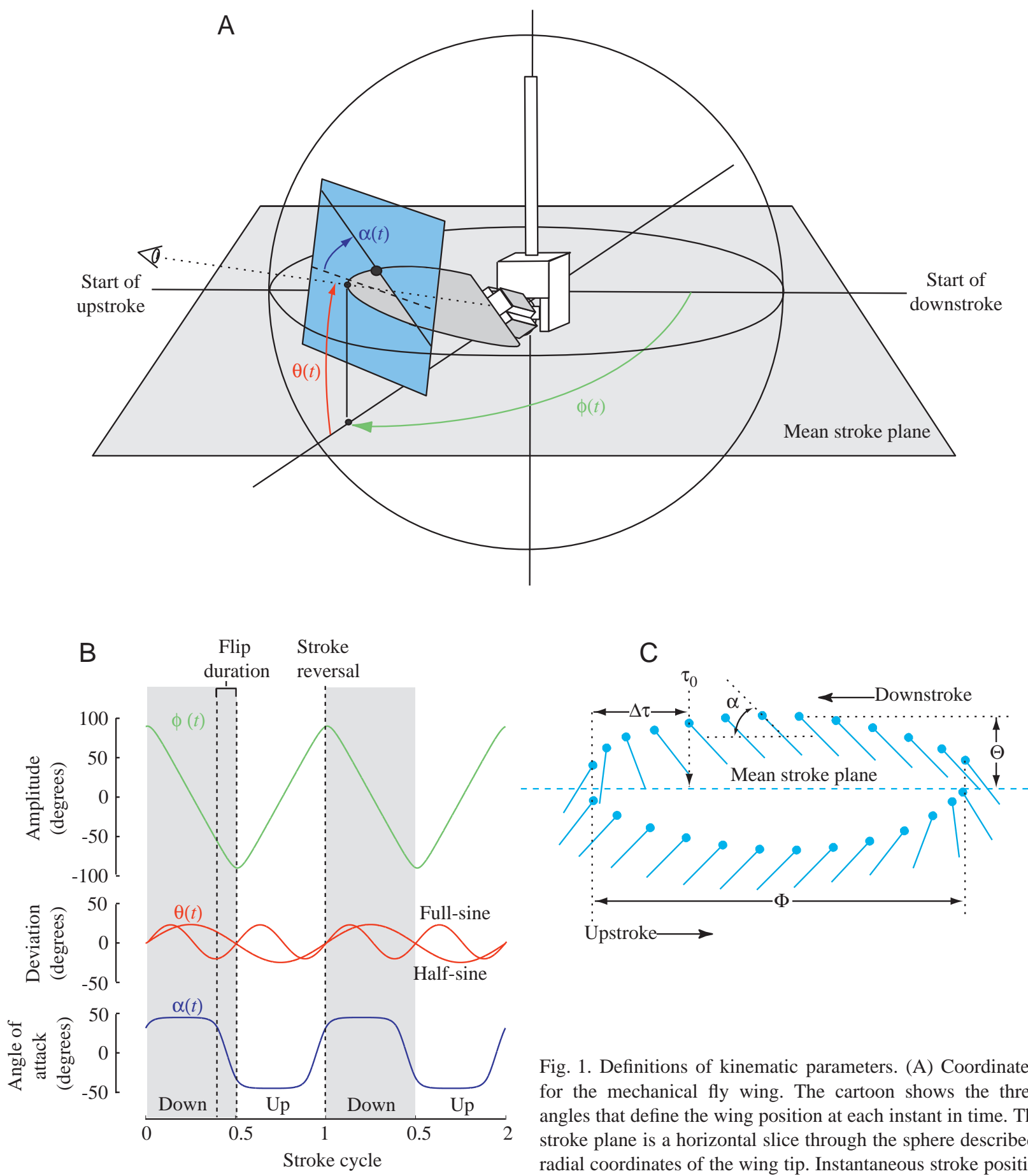

Fig. 1. Definitions of kinematic parameters. (A) Coordinate system for the mechanical fly wing. The cartoon shows the three Euler angles that define the wing position at each instant in time. The mean stroke plane is a horizontal slice through the sphere described by the radial coordinates of the wing tip. Instantaneous stroke position, $\phi(t)$, is defined as the angular position of the wing in the mean stroke plane, measured from dorsal reversal (start of downstroke) to ventral reversal (start of upstroke). Instantaneous stroke deviation, $\theta(t)$, is defined as the angle that the base-to-tip line on the wing makes with the mean stroke plane. A plane that is normal to the base-to-tip line of the wing (shown in blue) cuts through the wing at the wing chord, shown here as a line with a filled circle denoting the leading edge. The instantaneous angle of attack, $\alpha(t)$, is the angle that the wing chord makes with the tangent of the wing's trajectory. (B) Sample wing kinematics plotted over two complete cycles. Grey and white backgrounds mark downstroke and upstroke, respectively. Stroke position (green) follows a smoothed triangular waveform. Stroke deviation (red) varies as either a half or full sinusoid in each half-stroke. Half-sine variation yields an 'oval' tip trajectory (shown in C), whereas full-sine variation yields a 'figure-of eight' tip trajectory. Angle of attack (blue) varies as a trapezoidal function. The shape of the function is determined by setting the starting point of the flip, $\tau_{0}$, and flip duration, $\Delta \tau$. (C) Schematic diagram of the six parameters that were varied in the experiments: total stroke amplitude, $\Phi$, maximum stroke deviation, $\Theta$, mid-stroke angle of attack, $\alpha$, flip start, $\tau_{0}$, flip duration, $\Delta \tau$, and the shape of the wing tip trajectory. This cartoon represents a two-dimensional projection of a three-dimensional kinematic pattern, as if viewed within the blue plane in A. The broken blue line shows the mean stroke plane. This representation of the stroke is repeated throughout the paper. 
parameter is zero by definition, since their body velocity is zero. The free-flight cruising velocity of $D$. melanogaster is approximately $20 \mathrm{~cm} \mathrm{~s}^{-1}$ (David, 1978), while the mean velocity of the wing tip is $280 \mathrm{~cm} \mathrm{~s}^{-1}$ (Lehmann and Dickinson, 1997). Thus, even while flying forwards, the reduced frequency parameter is less than 0.1 , indicating that the effect of freestream velocity on force generation should be of secondary importance to the velocity generated by flapping. For these reasons, our experiments in still fluid, which match the hovering case, should also serve as a fair approximation for moderate forward speeds. Thus, the reduced frequency parameter is not significantly different for cruising D. melanogaster and our static, hovering model fly. To obtain the correct range of Reynolds numbers, we used an isometrically enlarged wing planform of an actual $D$. melanogaster wing to ensure that the shape parameters (Ellington, 1984a) were identical to those of $D$. melanogaster. Using available data on D. melanogaster morphology and kinematics (Lehmann and Dickinson, 1997), we estimated that a wing length of $0.25 \mathrm{~m}$, a surface area of the wing pair of $0.0334 \mathrm{~m}^{2}$ (or $0.0167 \mathrm{~m}^{2}$ for a single wing), a kinematic viscosity of $120 \mathrm{cSt}$ and a wingbeat frequency of $0.168 \mathrm{~Hz}$ would allow us to achieve Reynolds numbers in the same range as those of $D$. melanogaster.

\section{Stroke kinematics}

In the absence of wing deformation, the kinematics of the wings may be uniquely described by specifying the time course of three angles: stroke position, $\phi(t)$, angle of attack, $\alpha(t)$, and stroke deviation, $\theta(t)$ (Fig. 1B). In all experiments, the angular position of the wing within the stroke plane was described by a triangular waveform, which maintains a constant translational velocity throughout each half-stroke. The waveform was smoothed to minimize inertial accelerations during stroke reversal and to match more closely published stroke kinematics from a variety of insects (Ellington, 1984b; Zanker, 1990a). For smoothing, we filtered the triangular waveform using a zero-phase-delay low-pass two-pole Butterworth filter with a cut-off frequency equal to 10 times the stroke frequency of $0.17 \mathrm{~Hz}$. The peak-to-peak amplitude of the stroke angle waveform could be varied in each experiment. The angle of attack was described by a trapezoidal wave function, which maintained a constant angle of attack during each half-stroke and constant rotational velocity during stroke reversal. The shape of this waveform in each experiment was determined by setting the mid-stroke angles of attack during the upstroke and downstroke and by specifying the starting and stopping points for wing rotation. The resulting function was then smoothed using a low-pass filter with identical characteristics to that used for the stroke position waveform. We used two functions to describe stroke deviation: an 'oval' pattern in which the wing tip deviated from the stroke plane according to a half-sinewave per stroke period and a 'figure-of-eight' pattern in which the stroke deviation varied as a full sine-wave. These patterns were chosen because they roughly approximate patterns described for a variety of insects (Ellington, 1984b; Zanker, 1990a).
To create the kinematic patterns used in this study, we varied any or all of six parameters: (i) the stroke amplitude, (ii) the mid-stroke angle of attack during upstroke and downstroke, (iii) the timing of wing rotation at dorsal and ventral reversal, (iv) the duration of the stroke reversal, (v) the shape of the wing tip trajectory ('oval' or 'figure-of-eight') and (vi) the angular deviation from the mean stroke plane during the upstroke and downstroke (Fig. 1C). In most of the experiments, the deviation amplitude was set to zero, such that the wing tip remained within the stroke plane throughout the cycle. Under these conditions, the kinematics of the wing stroke were symmetrical such that the upstroke and downstroke were mirror images of one another. Only in trials using 'oval' stroke deviations were the kinematics of the two strokes not identical. The frequency of the wing stroke $(0.17 \mathrm{~Hz})$ remained constant in all experiments, as did the upstroke-to-downstroke duration ratio, which was fixed at 1 . We constructed the kinematic patterns using a custom-designed MATLAB program (Mathworks) to convert the angular trajectories into a series of stepper motor commands.

\section{Force measurements}

Signals from the two-dimensional sensor were acquired using a National Instruments data-acquisition board (model BNC 2090) in a PC running custom-designed software written in MATLAB. Data were filtered on-line with an active fourpole Bessel filter with a cut-off frequency of $10 \mathrm{~Hz}$ and off-line with a zero-phase-delay low-pass digital Butterworth filter with a cut-off frequency of $3 \mathrm{~Hz}$, which was 17.6 times the wing stroke frequency. Apart from increasing the highfrequency components resulting from motor jitter, increasing the cut-off frequency of the filter did not alter the time course of the force traces.

Each experiment consisted of one burst of four consecutive wing strokes following pre-programmed kinematics. The wing begins the first downstroke in still fluid, whereas during the subsequent strokes it moves through a wake created by the preceding strokes. As a result, the time course of forces generated during the first stroke is markedly different from those of subsequent strokes. For this reason, the data from the first stroke were excluded from this analysis, while those from the three subsequent strokes were averaged. Thus, each presented trace represents an average of three force records. After subtracting gravitational forces, the forces measured from the normal and parallel channels were transformed into lift, drag, thrust and radial components.

\section{Added mass}

The measured force at the wing base consists of gravitational, inertial and aerodynamic components. The gravitational contribution of the sensor and wing mass to the total force signal was easily calculated and subtracted from the measured force traces. The inertial components represent the acceleration forces on the mass of the sensor and wing as well as the added mass of the fluid around the wing. To examine the contribution of the inertial effects of the wing mass and 
sensor, we replaced the wing surface with an aerodynamically neutral inertial model of the wing. The aerodynamically neutral model was essentially a brass knob with the same mass and center of mass inside the oil as the Plexiglas wing. Because of its low surface area, the brass knob generated negligible aerodynamic forces compared with the Plexiglas wing. For any arbitrary kinematic pattern, the resulting force traces for the brass knob could be entirely accounted for by gravity. Thus, the inertial forces generated by flapping this brass model, and therefore the Plexiglas wing, were negligible and have been ignored. Compared with gravity and wing inertia, the noncirculatory forces due to added mass are more difficult to measure because the fluid acceleration induced by a moving wing changes dynamically as the wing rotates, decelerates or accelerates (Daniel, 1984).

To estimate the magnitude of added mass, we used an approximation derived for motions of an infinitesimally thin two-dimensional plate in an inviscid fluid (Sedov, 1965). Using blade element method, we adapted it to the case of a three-dimensional wing rotating around an axis located at onequarter chord length from the leading edge. The force contribution normal to the wing surface due to the added mass inertia is given by:

$$
\begin{aligned}
& F_{\mathrm{a}, \mathrm{N}}=\rho \frac{\pi}{4} R^{2} \bar{c}^{2}(\ddot{\phi} \sin \alpha+\ddot{\phi} \dot{\alpha} \cos \alpha) \int_{0}^{1} \hat{r} \hat{c}^{2}(\hat{r}) \mathrm{d} \hat{r}- \\
& \ddot{\alpha} \rho \frac{\pi}{16} \bar{c}^{3} R \int_{0}^{1} \hat{c}^{2}(\hat{r}) \mathrm{d} \hat{r},
\end{aligned}
$$

where $\rho$ is the fluid density, $R$ is the wing length, $\bar{c}$ is the mean chord length, $\hat{r}$ and $\hat{c}(\hat{r})$ are the non-dimensional radial position along the wing and non-dimensional chord length, respectively (for nomenclature, see Ellington, 1984a), $\phi$ is the angular position of the wing and $\alpha$ is the angle of attack. Using equation 1 , we calculated an estimate of added mass inertia for each set of kinematics. As illustrated by the representative trace in Fig. 2, the absolute contribution of added mass to net forces on the wing was quite small in all cases. Further, by comparing equation 1 and equation 3 (see below), it can be seen that the added mass forces $\left(\approx \rho R^{2} \bar{c}^{2}\right)$ scale in proportion to aerodynamic forces $\left(\approx \rho R^{3} \bar{c}\right)$ for geometrically similar wings. Thus, for identical kinematics and geometry, added mass will have the same physical effect on a model wing as on the wing of a fly, provided that the Reynolds number is the same. Because both added mass and aerodynamic contributions are biologically relevant, we chose not to subtract the inertial estimates from the presented force traces.

\section{Experimental procedures}

To examine the influence of the six kinematic variables on aerodynamic forces, we divided our analysis into three sets of experiments. First, we held the values of flip timing (symmetrical around stroke reversal), flip duration (16\% of stroke cycle period) and stroke deviation $\left(0^{\circ}\right)$ constant, while we systematically varied stroke amplitude and angle of attack.

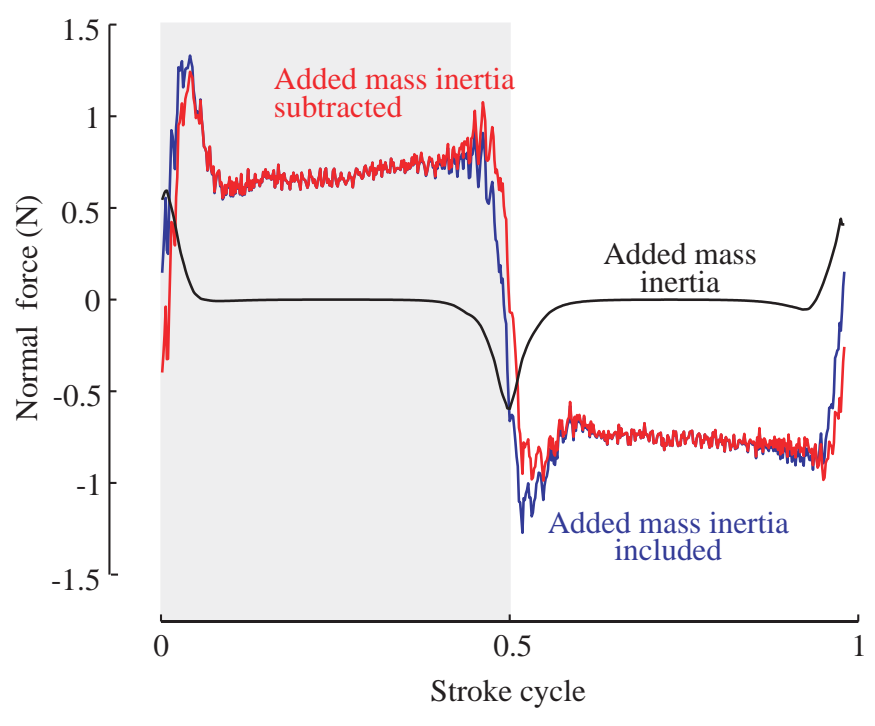

Fig. 2. Contribution of added mass inertia to total measured aerodynamic force. The traces shown were taken from a representative kinematic pattern (stroke amplitude $\Phi=180^{\circ}$, maximum stroke deviation $\Theta=0$, angle of attack $\alpha=45^{\circ}$, flip start, $\tau_{0}=0.05$, flip duration $\left.\Delta \tau=-0.1\right)$. The blue trace represents the total normal force measured on the wing. The static gravitational component has been subtracted. The black trace represents the added mass inertia estimated using equation 1 . Added mass inertia is zero throughout most of the stroke because the linear velocity of the wing is constant. The red trace represents the total measured force after subtracting added mass inertia. The contribution of added mass inertia to the measured aerodynamic forces is small, as indicated by the similarity of the red and blue traces.

For each amplitude from 60 to $180^{\circ}$ in $20^{\circ}$ increments, we varied the angle of attack at mid-stroke from 0 to $90^{\circ}$ in steps of $10^{\circ}$. From the results of this $7 \times 10$ set of experiments, we determined the combination of stroke amplitude and angle of attack that generated maximum mean lift. Using these locally optimizing values of stroke amplitude and angle of attack, we systematically varied the values of flip start and flip duration. Values for flip start indicate when in the stroke cycle the wing begins to rotate relative to stroke reversal and are expressed as a fraction of total cycle time (Fig. 1C). Thus, a value of -0.5 indicates that the wing begins rotation $50 \%$ of a stroke period prior to stroke reversal, whereas a flip start value of 0 indicates that the wing begins rotation at the instant of stroke reversal. Values of flip duration, the total time it takes the wing to complete wing rotation, are also represented as a fraction of total cycle time. Thus, a value of 0.2 indicates that a flip requires $20 \%$ of the stroke cycle to complete. In these experiments, we set the flip to start at various points within the stroke from -0.5 to 0 in steps of 0.05 . For each value of flip start, we varied the flip duration from 0.1 to -0.5 , also in steps of 0.05. Flip timing, $\tau_{\mathrm{f}}$, which describes when the mid-point of a flip occurs within the stroke, may be calculated from:

$$
\tau_{\mathrm{f}}=\tau_{0}+0.5 \Delta \tau,
$$

where $\tau_{0}$ is flip start and $\Delta \tau$ is flip duration. As before, we 


\section{S. P. SANE AND M. H. Dickinson}

determined the values of flip timing and flip duration that maximized mean lift within the $11 \times 9$ sets of trials. In the third set of experiments, we explored how aerodynamic forces varied with changes in deviation from the mean stroke plane using the values of stroke amplitude, angle of attack, flip timing and flip duration that maximize mean lift. In a set of 22 experiments, the total stroke deviation was varied from $-50^{\circ}$ $\left(-25^{\circ}\right.$ during the downstroke, $+25^{\circ}$ during the upstroke) to $+50^{\circ}\left(+25^{\circ}\right.$ during the downstroke, $-25^{\circ}$ during the upstroke) in steps of $10^{\circ}$. The deviation followed either a half-sine per stroke or a full-sine per stroke time course, yielding 'oval' and 'figure-of-eight' trajectories, respectively (Fig. 1B).

\section{Data analysis}

Since the conventions for lift and drag existing in current aerodynamic literature address non-flapping and primarily two-dimensional kinematic patterns, it is necessary to modify them slightly for three-dimensional motions. These modifications are based on the following two considerations. First, it is convenient to use a reference frame based on the insect body rather than its wing so that the measurements relate directly to free-flight studies. Second, the standard convention should apply when the kinematics reduce to two-dimensional motion. With these constraints in mind, we adopted the following convention: net aerodynamic force, defined as the total force on the wing, is resolved into three components: lift, drag and radial force. In hovering flight, lift must offset the gravitational force on the animal's body mass. Hence, we define lift as the component of the net aerodynamic force perpendicular to the mean stroke plane of the wing regardless of its actual instantaneous trajectory. Since the mean stroke plane was horizontal in all experiments, lift is always the vertical force component. Drag is defined as the force component in the horizontal direction, opposing the wing movement. The radial component accounts for the remaining force component in the horizontal plane. For motions with no stroke deviation, these definitions reduce to the standard convention: lift is orthogonal to drag, and the radial component vanishes. With stroke deviation, the total normal pressure force consists of orthogonal vertical and radial components, each orthogonal to the drag vector in the horizontal direction.

From the forces on each wing, we calculated the corresponding mean force coefficients using an equation derived from blade element theory (Ellington, 1984c; Dickinson et al., 1999):

$$
\overline{C_{\mathrm{F}}}=\frac{8 \bar{F}}{\rho \Phi^{2} n^{2} R^{3} \overline{\bar{c}} \overline{\mathrm{d} \hat{\phi} / \mathrm{d} \hat{t})^{2}} \hat{r}_{2}^{2}(S)},
$$

where $\bar{F}$ is the magnitude of a specific force component (lift, drag, radial, total) averaged over the stroke, $\Phi$ is stroke amplitude, $n$ is wing beat frequency, $\overline{\mathrm{d}} \hat{\phi} / \mathrm{d} \hat{t}$ is the mean nondimensional angular velocity of the wing and $\hat{r}_{2}^{2}(S)$ is the nondimensional second moment of wing area. The radial force component changes sign when the wing crosses the mean stroke plane. As a result, in the 'oval' as well as the 'figure- of-eight' patterns, the mean radial coefficient often averages to zero and is uninformative. For this reason, we base our measurement of the average radial force coefficient on the absolute values. Lift-to-drag ratios were calculated by dividing the mean lift coefficient by the mean drag coefficient. Similarly, radial-to-drag ratios were obtained by dividing the mean absolute radial coefficients by the mean drag coefficients. To calculate the ratio of mean lift to profile power, we estimated mean profile power, $\overline{P_{\text {pro }}}$, based on the timeaveraged product of instantaneous drag, $D(t)$, and instantaneous velocity, $v_{\text {wing }}(t)$ :

$$
\overline{P_{\text {pro }}}=n \int_{t=0}^{t=T} D(t) \vee_{\text {wing }}(t) \mathrm{d} t,
$$

where $T$ is the stroke period. This calculation of mean profile power ignores the power required to rotate the wing in place. Mean lift $\bar{L}$ was calculated as the time average of instantaneous lift throughout the stroke.

Measures of the quasi-steady-state translational force coefficients $C_{\mathrm{L}, \mathrm{t}}$ and $C_{\mathrm{D}, \mathrm{t}}$ were derived from $180^{\circ}$ sweeps of wing motion with fixed angles of attack, as described elsewhere (Dickinson et al., 1999). The equations that best fit measured translational force coefficients as functions of angle of attack, $\alpha$, for the model wing are (Dickinson et al., 1999):

$$
C_{\mathrm{L}, \mathrm{t}}=0.225+1.58 \sin (2.13 \alpha-7.2) \text {, }
$$

and

$$
C_{\mathrm{D}, \mathrm{t}}=1.92-1.55 \cos (2.04 \alpha-9.82) \text {. }
$$

These equations are used to generate quasi-steady translational estimates for comparison with measured values.

\section{Results \\ The effects of stroke amplitude and angle of attack}

Fig. 3A-H shows eight sample records taken from the full set of 70 trials to illustrate how the magnitude and time course of aerodynamic forces vary with stroke amplitude and angle of attack. These panels also show the lift and drag forces reconstructed from a quasi-steady model based on measured translational force coefficients. The quasi-steady predictions provide a conservative estimate of forces that would result from a stable leading edge vortex during translation. Any deviation of a measured trace from the quasi-steady estimate represents an unsteady effect that is not active during translation. In all records, the net aerodynamic force is nearly perpendicular to the wing surface throughout the stroke, indicating that shear forces measured parallel to the wing surface are much smaller than the aerodynamic pressure forces that must act normal to the thin flat wing. The force trajectories during the downstroke and upstroke, though very similar, are not precisely identical because of small asymmetries in the stroke pattern introduced by the gearbox of the model. As suggested by the reconstructions in Fig. 2, the transient undershoots in the drag traces at stroke reversal $(t=0,0.5)$ are explained in part by added mass inertia. 
Fig. 3. Sample instantaneous forces for various combinations of total stroke amplitude $\Phi$ and mid-stroke angle of attack $\alpha$. In each case, the wing rotation parameters were kept constant (flip duration $\Delta \tau=0.16$, flip start $\tau_{0}=-0.08$, flip timing $\tau_{\mathrm{f}}=0$ ). Each panel (A-H) shows a plot of measured drag (solid red line), the quasi-steady estimate of drag (broken red line), measured lift (solid blue line) and the quasisteady lift (broken blue line). Since the radial forces were zero for all these kinematic patterns, they are not plotted. A twodimensional diagram of the wing kinematics is plotted above each set of traces using the convention described in Fig. 1C. The wing chord is shown in light blue, and the superimposed black vector indicates the magnitude and direction of the instantaneous aerodynamic force. For convenience, the kinematic values of stroke amplitude and angle of attack used in each trial are printed in the upper left of each panel. Values for the measured mean force coefficients $\left(\overline{C_{\mathrm{D}}}\right.$ and $\left.\overline{C_{\mathrm{L}}}\right)$ are printed adjacent to each set of traces. Axis labels given in A apply to all panels. (A,B) Forces generated at a $90^{\circ}$ angle of attack with no wing rotation $\left(\alpha=90^{\circ}\right)$ for a short stroke amplitude (A, $\Phi=60^{\circ}$ ) and a long stroke amplitude (B, $\Phi=180^{\circ}$ ). Note the enormous transients in drag at the start of each stroke due to wake capture. $(\mathrm{C}, \mathrm{D})$ Forces generated at a $50^{\circ}$ angle of attack for a short stroke amplitude $\left(\mathrm{C}, \Phi=60^{\circ}\right)$ and a long stroke amplitude (D, $\Phi=180^{\circ}$ ). The contribution of rotational circulation is apparent at the end of each stroke. (E,F) Forces generated at a $30^{\circ}$ angle of attack for a short stroke amplitude (D, $\Phi=60^{\circ}$ ) and a long stroke amplitude $\left(\mathrm{F}, \quad \Phi=180^{\circ}\right) . \quad(\mathrm{G}, \mathrm{H})$ Forces generated at a $0^{\circ}$ angle of attack for a short stroke amplitude (G, $\Phi=60^{\circ}$ ) and a long stroke amplitude $\left(\mathrm{H}, \Phi=180^{\circ}\right)$.
A
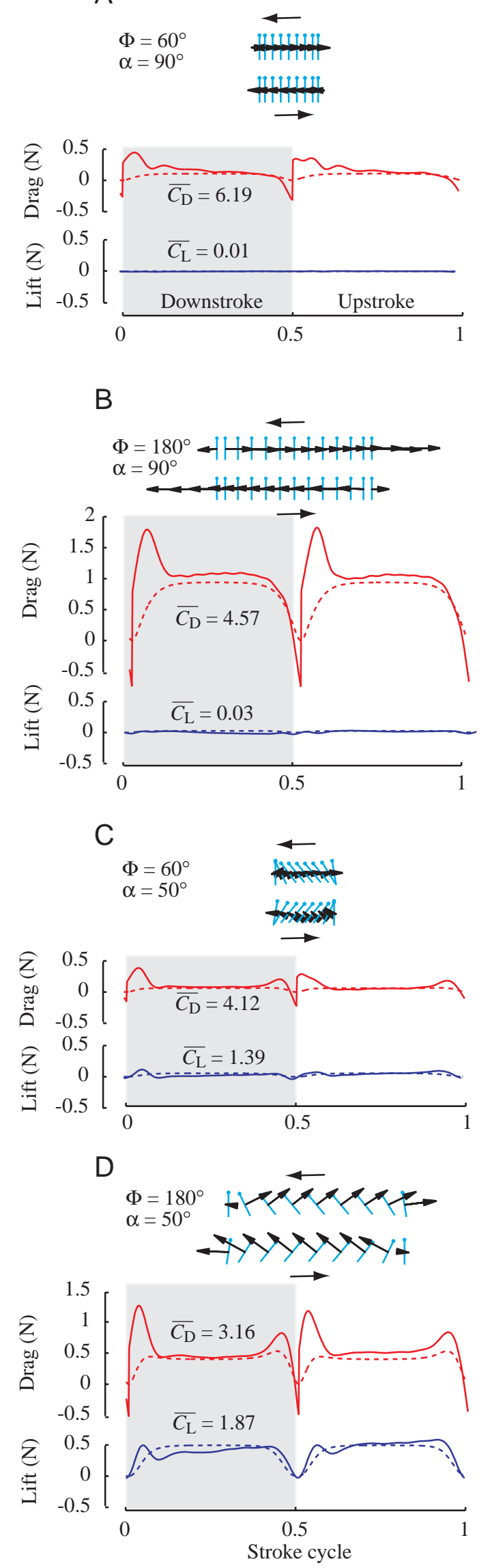

E

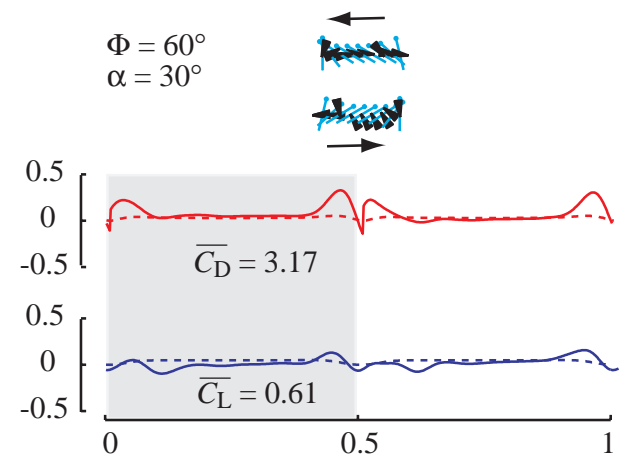

$\mathrm{F}$

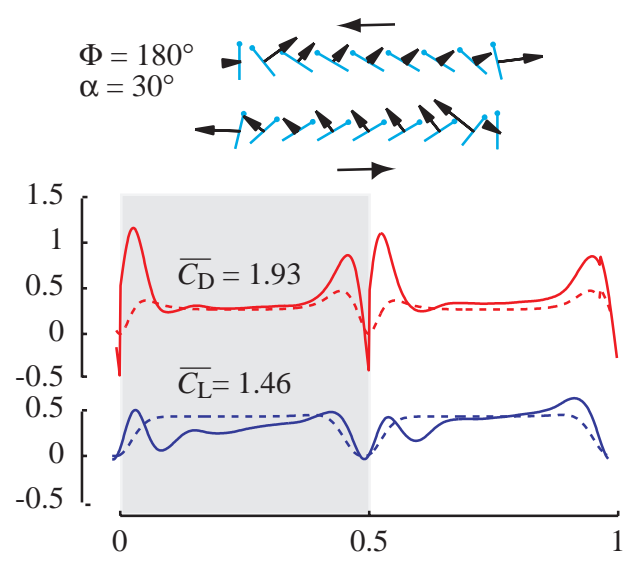

G
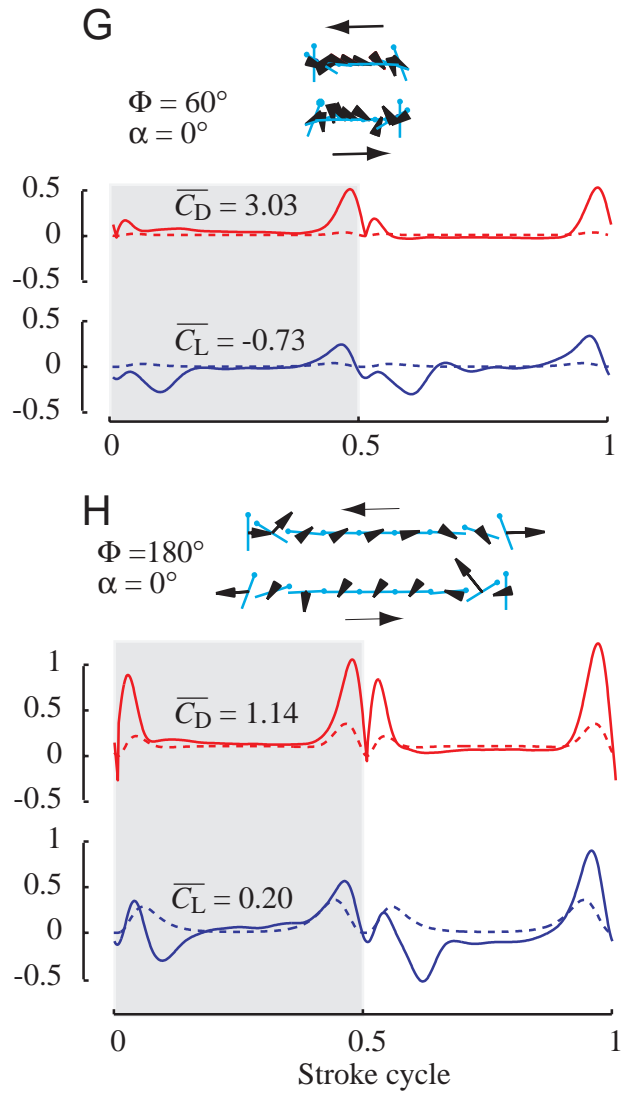


\section{S. P. SANE AND M. H. Dickinson}

The measured force trajectories display prominent peaks at the beginning and end of each translational stage that are absent or smaller in the quasi-steady predictions. In isolating the mechanisms responsible for these transient effects, it is useful to consider cases in which there is no wing rotation, because this removes the potential contribution of rotational circulation. For example, in Fig. 3A,B, the wing translates back and forth at a $90^{\circ}$ angle of attack without rotating. At the start of each stroke, measured drag far exceeds the quasi-steady predictions. The fact that there is no corresponding dip in drag at the end of translation indicates that the prominent drag transient is not due to acceleration of inertial mass. This interpretation is consistent with estimates of added mass inertia using equation 1 (data not shown). Instead, the elevation in drag at the start of each stroke in Fig. 3A,B is probably due to wake capture as the wing intercepts the fluid flow field induced by the shed vorticity of the previous stroke.

The influence of rotational circulation is most easily seen when the angle of attack during the upstroke and downstroke is zero (Fig. 3G,H). Under these conditions, the vorticity generated during the translational phase of the stroke is minimal and, thus, the magnitude of the wake capture effect should be small. The influence of the wake is not entirely absent, however, because the process of rotation generates and sheds vorticity through which the wing must translate at the start of each stroke. Further, the vorticity created by rotation is particularly strong when the translational angle of attack is zero, because the wing must flip over by $180^{\circ}$ during stroke reversal, making the angular velocity of the wing particularly large. At the end of the each stroke in Fig. 3G,H, the influence of rotational circulation is manifest as a transient increase in lift and drag that exceeds the quasi-steady prediction. After stroke reversal, the continuing rotation of the wing generates a pressure force with opposite polarity, resulting in negative lift. The time course of this rotational effect is complicated by the presence of an added mass inertia and a modest amount of wake capture at the start of each stroke. The influence of these multiple mechanisms is manifest by the positive peak in lift immediately following stroke reversal due to wake capture, which is followed by the negative peak due to rotational circulation. The pattern of an early positive peak in lift followed by a later negative peak is seen throughout the traces in Fig. 3.

The rest of the traces in Fig. 3 illustrate the complex interactions among delayed stall, rotational circulation and wake capture that result from changes in angle of attack and stroke amplitude. At angles of attack of 30 and $50^{\circ}$, the wing generates lift throughout the stroke due to delayed stall (Fig. 3C-F). The influence of rotational lift is reduced as the angle of attack increases, however, because the wing flips over a smaller arc with lower angular velocity. This effect can be seen by comparing the relative magnitude of the force peaks at the end of each stroke in Fig. 3B,D,F,H. In contrast, the influence of wake capture is greater at higher angles of attack because the vorticity shed into the wake at the end of the stroke is stronger. This effect can be seen by comparing the relative
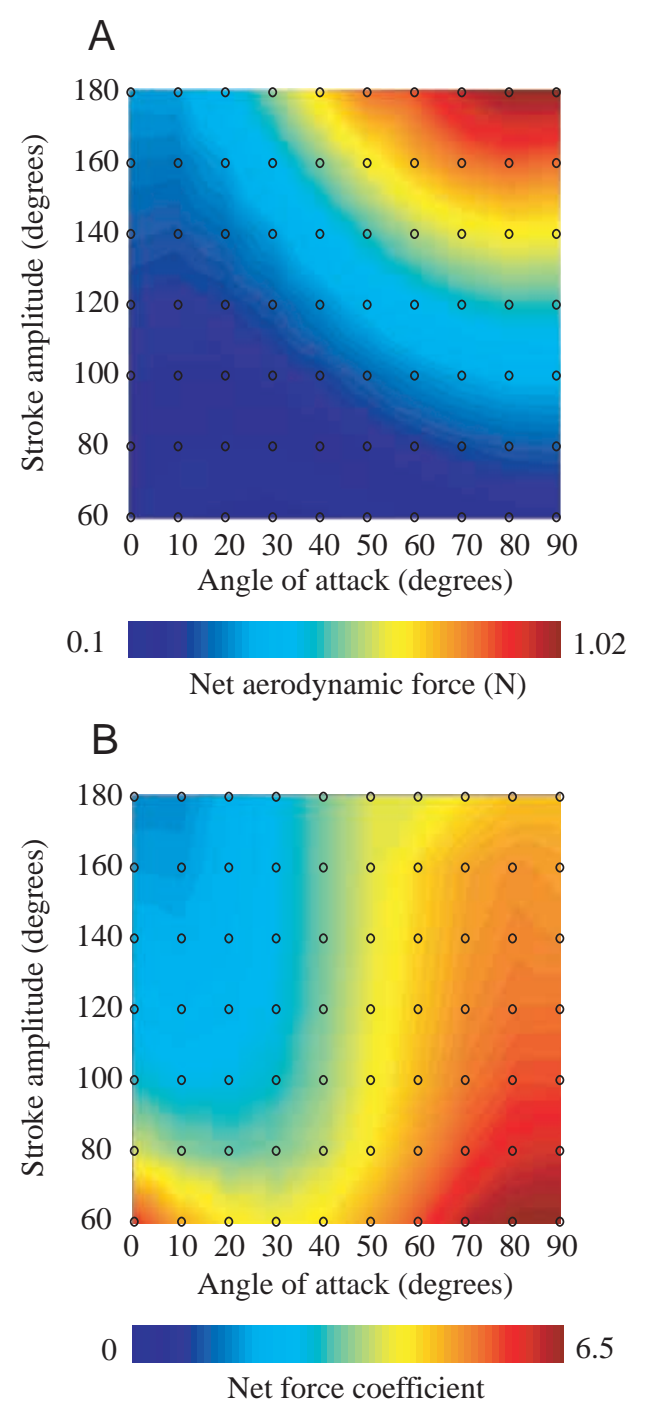

Fig. 4. Parameter maps of net aerodynamic force and net force coefficient as functions of stroke amplitude and mid-stroke angle of attack. For fixed values of wing rotation (flip duration $\Delta \tau=0.16$; flip start $\tau_{0}=-0.08$, flip timing $\tau_{\mathrm{f}}=0$ ), stroke amplitude was varied from 60 to $180^{\circ}$ and angle of attack was varied from 0 to $90^{\circ}$. In each diagram, the small open circles indicate the positions of actual measurements. Values between these measured points have been interpolated using a cubic spline. Values are encoded in pseudocolor according to the scales shown beneath each plot. This same format is used in Fig. 5, Fig. 7 and Fig. 10. (A) Net aerodynamic force, the vector sum of lift and drag, increases monotonically with increasing angle of attack and stroke amplitude. (B) Net aerodynamic force coefficient increases with angle of attack, but decreases with stroke amplitude.

size of the force transients at the start of each stroke in the same panels. Thus, changing stroke amplitude and angle of attack has a complex but interpretable influence on the magnitude of the different unsteady mechanisms. The kinematics that optimize the aerodynamic performance of the wing will reflect these complex interactions. The maximum mean lift-to-drag ratio (0.8) occurred at an angle of attack of $30^{\circ}$ and amplitude 


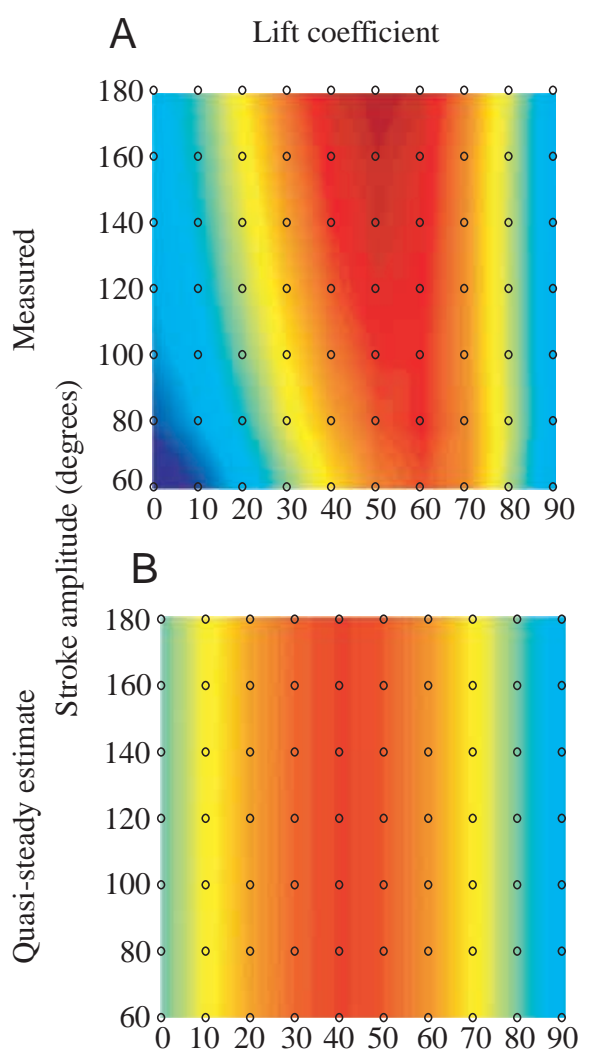

$-0.75$
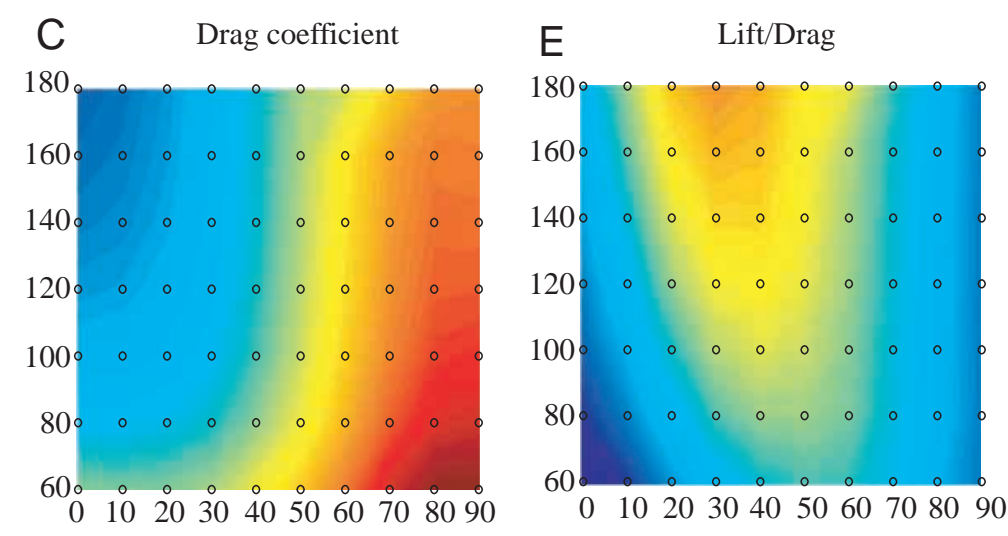

D

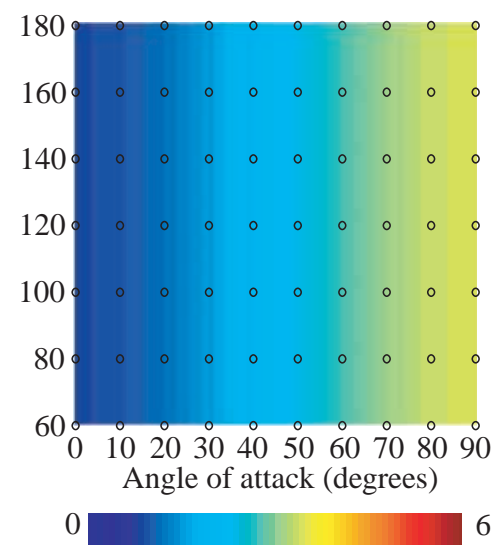

$\mathrm{F}$

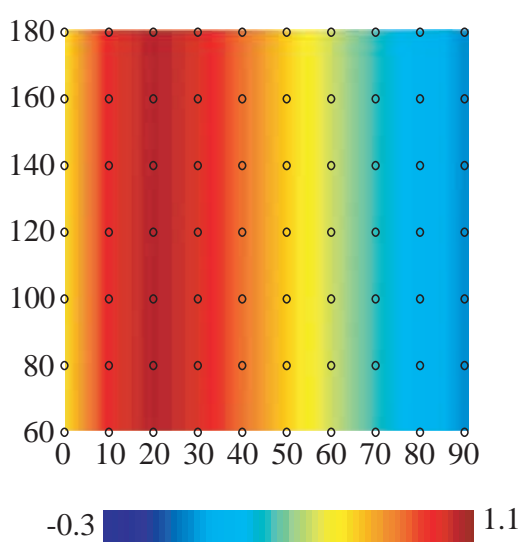

Fig. 5. Parameter maps of stroke-averaged lift coefficient, drag coefficient and lift-to-drag ratio as functions of angle of attack and stroke amplitude. The data are plotted as in Fig. 4. Axis labels given in A apply to all panels. Pseudocolor scales apply to both figures in a given column. The top panels (A,C,E) show maps of values measured from the mechanical model, and the bottom panels (B,D,F) show the corresponding values calculated for a translational quasi-steady model using empirically measured force coefficients. (A,B) Measured and quasi-steady values for mean lift coefficient. (C,D) Measured and quasi-steady values for mean drag coefficient. The quasi-steady predictions grossly underestimate the drag coefficient. (E,F) Measured and quasi-steady values for mean lift-to-drag ratio.

of $180^{\circ}$. The forces corresponding to this optimal condition are shown in Fig. 3F.

To provide a more comprehensive picture of how force production changes with kinematics, we calculated the mean lift, drag and net force coefficients averaged throughout the stroke and plotted them for all pairs of stroke amplitude and angle of attack values. Fig. 4A,B depicts the mean total aerodynamic force, $\overline{F_{\mathrm{N}}}$, and force coefficient, $\overline{C_{\mathrm{N}}}$, in pseudocolor maps. These two maps differ from one another because the mean force coefficient is normalized with respect to the square of stroke amplitude, which is a variable in these experiments. Thus, while $\overline{F_{\mathrm{N}}}$ increases with increasing stroke amplitude (Fig. 4A), $\overline{C_{\mathrm{N}}}$ decreases with increasing stroke amplitude (Fig. 4B). Both parameters rise with increasing angle of attack. The influence of stroke amplitude and angle of attack on the mean lift coefficient $\overline{C_{\mathrm{L}}}$ and the mean drag coefficient $\overline{C_{\mathrm{D}}}$ is shown in Fig. 5A,C. For a fixed stroke amplitude, $\overline{C_{\mathrm{L}}}$ exhibits a broad maximum ranging from 1.8 to 2.0 between angles of attack of 40 and $50^{\circ}$. As expected, $\overline{C_{\mathrm{D}}}$ rises monotonically with increasing angle of attack for any given value of stroke amplitude. It is worth noting that the range of $\overline{C_{\mathrm{D}}}$ values is much higher than has been previously reported for Drosophila virilis wings under steady-state conditions (Vogel, 1967) or estimated on the basis of Reynolds number $\left(C_{\mathrm{D}} \approx 0.7\right.$; Ellington, $\left.1984 \mathrm{c}\right)$. Although much less pronounced than the dependence on angle of attack, lift tends to rise, whereas drag falls, with increasing stroke amplitude.

The corresponding maps for the quasi-steady translational estimates, $\overline{C_{\mathrm{L}, \mathrm{t}}}$ and $\overline{C_{\mathrm{D}, \mathrm{t}}}$, are shown in Fig. 5B and Fig. 5D, respectively. In general, the measured force coefficients are greater than the predicted quasi-steady force coefficients. This discrepancy is particularly large for $C_{\overline{\mathrm{D}}}$. Further, at high stroke amplitude, the angle of attack that generates the maximum $\overline{C_{\mathrm{L}}}$ is shifted by approximately $10^{\circ}$ relative to the quasi-steady predictions and by as much as $20^{\circ}$ for the lower stroke amplitudes. The dependence of stroke amplitude on the measured lift and drag coefficients is not predicted by the quasi-steady estimates. The greater difference between measured and predicted values for smaller stroke amplitudes underscores the increased importance of rotational effects under these conditions. Fig. 5E indicates how the mean lift-todrag ratio, $\overline{C_{\mathrm{L}}} / \overline{C_{\mathrm{D}}}$, varies with angle of attack and stroke 
amplitude. The maximum value of $\overline{C_{\mathrm{L}}} / \overline{C_{\mathrm{D}}}(0.8)$ occurred at stroke amplitude of $180^{\circ}$ and an angle of attack of $30^{\circ}$. The corresponding quasi-steady-state estimates of $\overline{C_{\mathrm{L}}} / \overline{C_{\mathrm{D}}}$ are independent of stroke amplitude, with a maximum of 1.1 at an angle of attack of $20^{\circ}$. Thus, the quasi-steady model significantly overestimates aerodynamic efficiency and fails to account for its dependence on stroke amplitude.

\section{The effects of flip timing and flip duration}

In all subsequent experiments, we set the stroke amplitude to $180^{\circ}$ and the mid-stroke angle of attack to $45^{\circ}$, which are the values that maximized lift production in the first set of experiments. Next, we systematically varied the timing and duration of wing rotation to examine their effects on force production. Sample force traces selected from 99 pairs of flip start and flip duration are shown in Fig. 6A-H, with the corresponding values of flip timing, $\tau_{\mathrm{f}}$, given in the upper left corner of each panel. A comparison of Fig. 6A,C,D illustrates that a long-duration flip, $\Delta \tau=0.5$, can produce quite different forces depending on when the flip occurs. If the flip occurs symmetrically about the stroke reversal $\left(\tau_{\mathrm{f}}=0\right.$, Fig. $\left.6 \mathrm{~A}\right), \overline{C_{\mathrm{L}}}$ is quite large $\left(\overline{C_{\mathrm{L}}}=1.54\right)$ and the time course is well approximated by the quasi-steady predictions. An advanced flip $\left(\tau_{\mathrm{f}}=-0.25\right.$, Fig. 6C) results in very low mean lift $\left(\overline{C_{\mathrm{L}}}=0.36\right)$, but produces a fairly prominent wake capture peak. In contrast, a rotation after stroke reversal $\left(\tau_{\mathrm{f}}=+0.25\right.$, Fig. $\left.6 \mathrm{D}\right)$ results in mean negative lift $\left(\overline{C_{\mathrm{L}}}=-0.28\right)$ because of the adverse effects of rotational circulation following stroke reversal.

Fig. $6 \mathrm{~F}-\mathrm{H}$ shows the effects of a fast flip $(\Delta \tau=0.1)$ at different flip times. As flip duration decreases, the aerodynamic performance of the wing generally rises. Symmetrical and advanced flips yield nearly identical mean lift $\left(\overline{C_{L}}=1.9\right.$, symmetrical; $\overline{C_{\mathrm{L}}}=1.87$, advanced), whereas a delayed flip generates somewhat lower lift $\left(\overline{C_{\mathrm{L}}}=1.52\right)$. These differences are due primarily to the amount of lift produced at the start of each stroke. Early and symmetrical flips (Fig. 6F,G) result in a substantial wake capture peak at the start of translation. However, if the flip occurs very early in the stroke $\left(\tau_{\mathrm{f}}=-0.45\right.$, Fig. 6E), the wing translates through most of the stroke at negative angles of attack, leading to a large value of negative lift $\left(\overline{C_{\mathrm{L}}}=-1.41\right.$; Fig. $\left.6 \mathrm{E}\right)$. When rotation is delayed, the wake capture peak is missing, revealing two negative peaks at the start of translation, an early small peak due to added mass inertia and a later more prominent peak due to rotational circulation (Fig. 6H).

The maps of mean force coefficients as a function of flip duration and flip start are shown in Fig. 7A,C,E, with comparable quasi-steady, translational predictions shown in Fig. 7B,D,F. Flip timing, the non-dimensional time when the mid-point of the flip occurs, is indicated by the inclined parallel lines on each graph. Both $\overline{C_{\mathrm{L}}}$ and $\overline{C_{\mathrm{D}}}$ are strongly influenced by flip timing and duration. For example, at a flip duration of $0.1, \overline{C_{\mathrm{L}}}$ varies with flip timing from as low as -1.5 to as high as +2 . The comparable values of the quasi-steady translational estimate, $\overline{C_{\mathrm{L}, \mathrm{t}}}$, also vary, but over a smaller range (from -1 to 1.6).
For all values of flip timing, $\overline{C_{\mathrm{D}}}$ increases monotonically with flip duration (Fig. 7C). Measured values range from 2.6 and 4.1, representing a somewhat smaller variation than was seen with $\overline{C_{\mathrm{L}}}$. However, unlike the case with lift, the discrepancy between measured $\overline{C_{\mathrm{D}}}$ and the quasi-steady estimate, $\overline{C_{\mathrm{D}, \mathrm{t}}}$, is substantial. In addition to generally underestimating the magnitude of drag, the quasi-steady predictions fail to observe the local rise in drag along the -0.25 flip timing iso-line. Since the range of variation for drag is less than that for lift, the measured lift-to-drag ratio map resembles the lift coefficient map (Fig. 7A,C,E). Further, because the quasi-steady translational predictions underestimate lift and drag by approximately the same proportion, the predicted liftto-drag ratio map is quite similar to the measured map (Fig. 7E,F). The map for the net force coefficient (Fig. 7G) resembles the drag map (Fig. 7C), which is expected since the values for the mean drag coefficient are, on average, twice those for the lift coefficient at comparable points on the kinematic surface.

\section{The effects of stroke plane deviation}

Using kinematic values for stroke amplitude, angle of attack, flip duration and flip start that maximized lift production $\left(\Phi=180^{\circ}, \alpha=45^{\circ}, \Delta \tau=0.1\right.$ and $\left.\tau_{\mathrm{f}}=-0.05\right)$, we tested how forces vary with deviation from the mean stroke plane in a set of 22 experiments. The peak-to-peak magnitude of stroke deviation was varied from -50 to $+50^{\circ}$ in $10^{\circ}$ steps for both the halfsine ('oval') and full-sine ('figure-of-eight') patterns. It is worth noting that, in the oval pattern, an upward deviation at the start of the downstroke requires a downward deviation at the start of the upstroke and vice versa. This is not the case for the figure-of-eight pattern, in which the two half-strokes are mirror images of one another.

Fig. 8 shows a selection of force traces resulting from different patterns of stroke deviation. In general, the figure-ofeight pattern had a more profound influence on the magnitude and time course of force production than did the oval pattern. In both cases, however, the direction of stroke deviation at the start of each translational phase greatly influenced the magnitude of the force transient at the start of the stroke. For example, in the figure-of-eight pattern shown in Fig. 8C, each stroke begins with an upward motion, and the lift and drag transients at the start of each stroke are quite small. In contrast, the comparable kinematic pattern that starts with a downward motion (Fig. 8D) generates sizeable force peaks. A similar trend is seen in the oval patterns (Fig. 8A,B). The upstroke in Fig. 8A and the downstroke in Fig. 8B, which both start with a downward motion, are marked by sizeable force peaks at the start of translation, whereas the strokes that begin with upward motion are not. This dependence of the early force transient on the direction of deviation is explained in part by an increase in the aerodynamic angle of attack caused by the downward motion of the wing. However, the measured force peaks are much greater than the quasi-steady estimates, which take into account this effect, suggesting that there is a substantial wake effect at the start of each stroke. The influence of the wake is 
Fig. 6. Sample instantaneous forces for various combinations of flip start $\tau_{0}$ and flip duration $\Delta \tau$. In all kinematic patterns, stroke amplitude was $180^{\circ}$ and angle of attack was $45^{\circ}$. The format for each panel is that described for Fig. 3. As in Fig. 3, the radial forces for all these kinematics are zero and have not been plotted. (A) Forces generated with a slow flip $(\Delta \tau=0.5)$, symmetrical with respect to stroke reversal $\left(\tau_{0}=-0.25\right.$, flip timing $\left.\tau_{\mathrm{f}}=0\right)$. Under these conditions, the quasi-steady model (broken lines) accurately predicts measured lift, but not drag. (B) Forces generated with moderate flip duration $(\Delta \tau=0.25)$, advanced with respect to stroke reversal $\left(\tau_{0}=-0.25\right.$, $\left.\tau_{\mathrm{f}}=-0.125\right)$. With these kinematics, the augmentation of lift by rotational circulation and wake capture is evident. (C) Forces generated with a long, advanced flip $\left(\Delta \tau=0.5 ; \quad \tau_{0}=-0.5, \tau_{\mathrm{f}}=-0.25\right)$. This pattern of kinematics produced elevated drag due to wake capture at the start of each stroke. (D) Same kinematics as in C, but with a delayed flip $\left(\Delta \tau=0.5 ; \tau_{0}=0\right.$, $\left.\tau_{\mathrm{f}}=+0.25\right)$. The delay in flip timing causes a small decrease in mean drag, but an enormous decrease in lift. (E-H) The influence of rotational timing on a shortduration flip. (E) Forces generated by a short flip advanced by almost a full half-cycle with respect to stroke reversal $\left(\Delta \tau=0.1 ; \tau_{0}=-0.5\right.$, $\left.\tau_{\mathrm{f}}=-0.45\right)$. Note that the angle of attack is negative during most of translation because the wing flips much too soon. As a consequence, the pattern generates negative lift. (F) Forces generated by a slightly advanced short flip $(\Delta \tau=0.1$; $\left.\tau_{0}=-0.1, \quad \tau_{\mathrm{f}}=-0.05\right)$. This nearoptimal pattern augments lift by both rotational mechanisms. (G) Forces generated by a short symmetrical flip $\left(\Delta \tau=0.1 ; \tau_{0}=-0.05\right.$, $\tau_{\mathrm{f}}=0$ ). $(\mathrm{H})$ Forces generated by a slightly delayed short flip $(\Delta \tau=0.1$; $\left.\tau_{0}=0, \tau_{\mathrm{f}}=0.05\right)$. The small delay of 0.05 decreases the mean lift coefficient by $20 \%$ compared with the symmetrical case shown in $\mathrm{G}$. $\overline{C_{\mathrm{D}}}$, mean drag coefficient; $\overline{C_{\mathrm{L}}}$, mean lift coefficient.
A

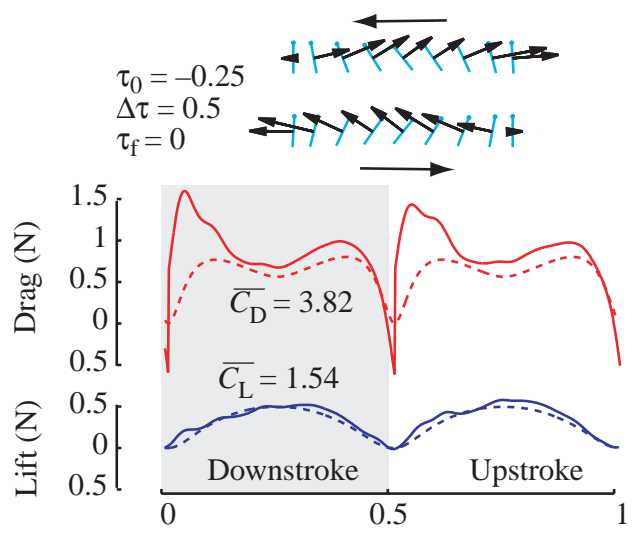

B
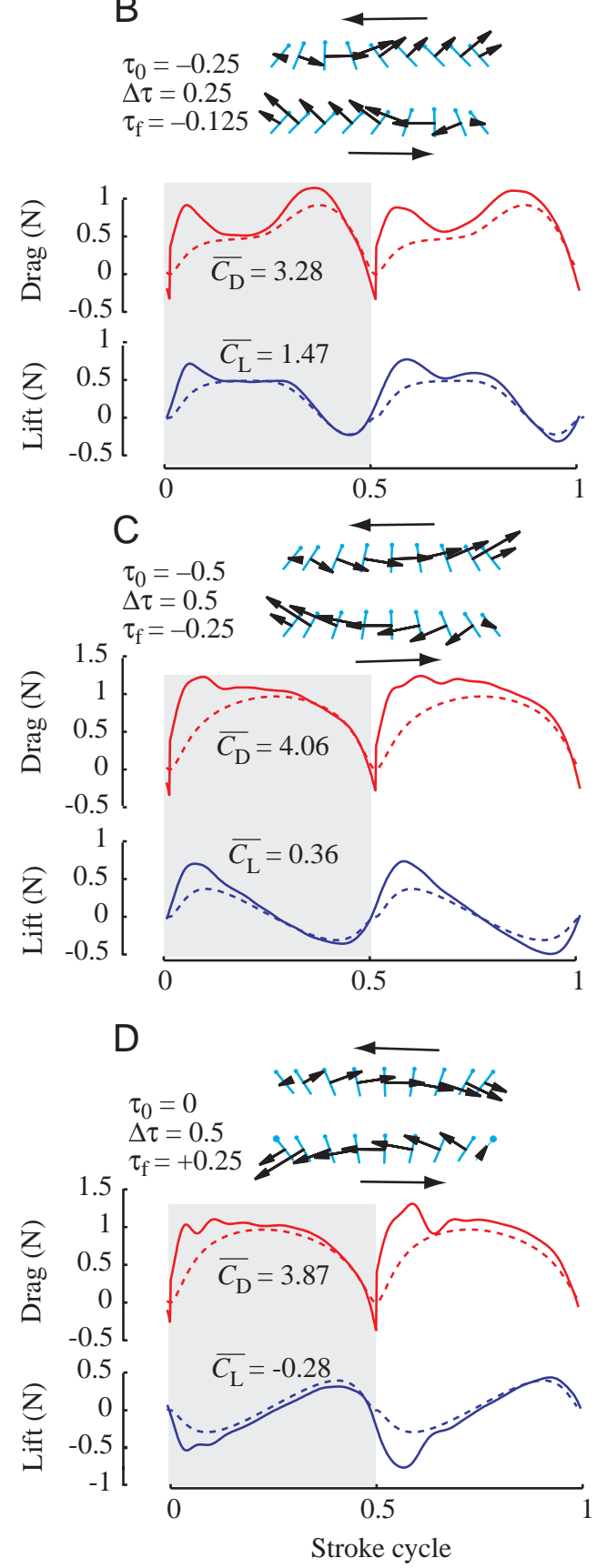

E
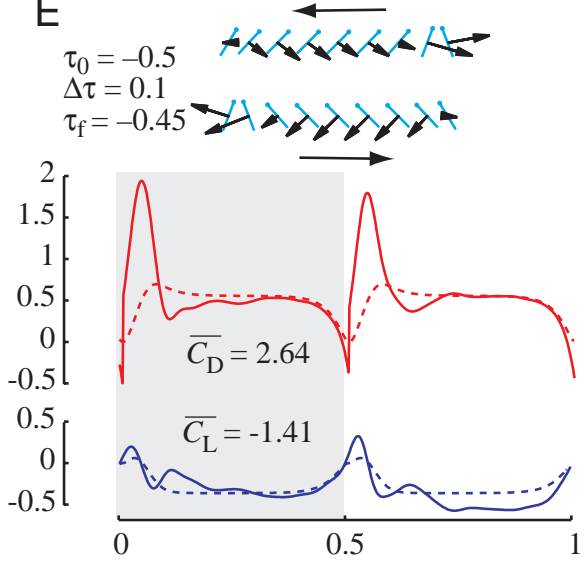

$\mathrm{F}$

$\tau_{0}=-0.1$

$\Delta \tau=0.1$

$\tau_{\mathrm{f}}=-0.05$

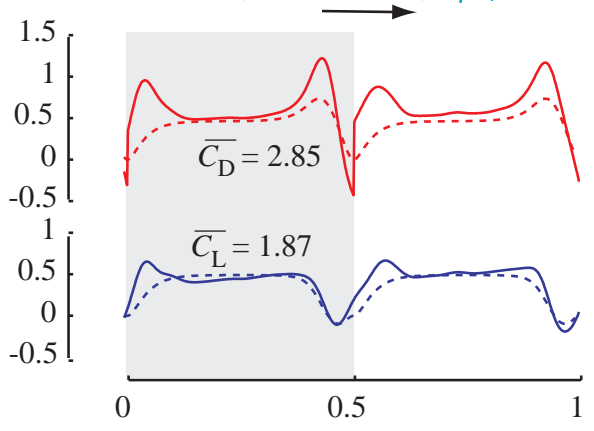

G
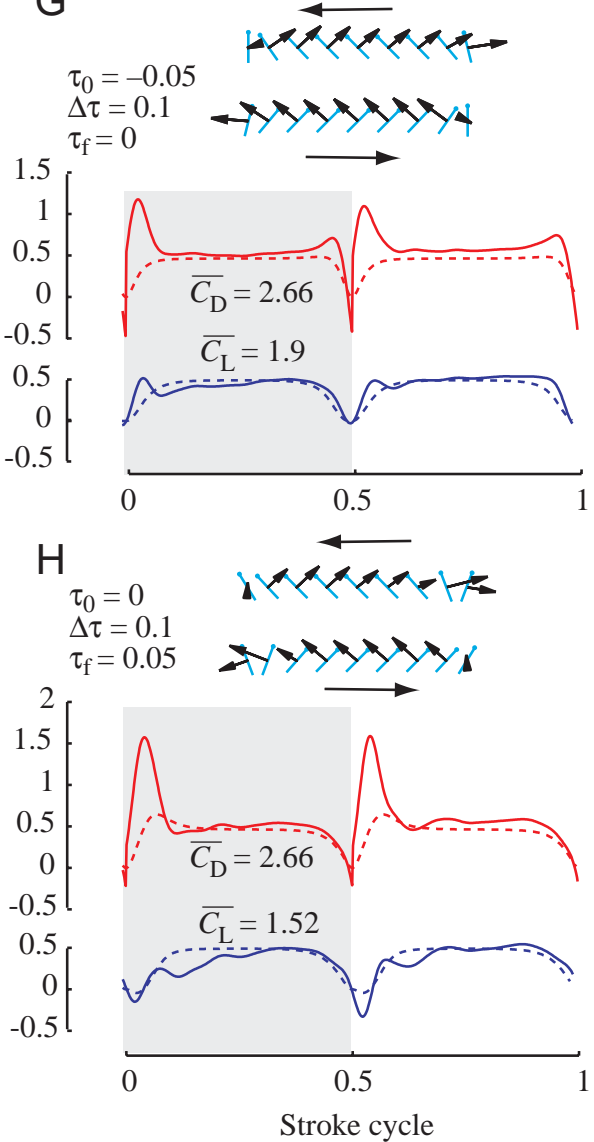

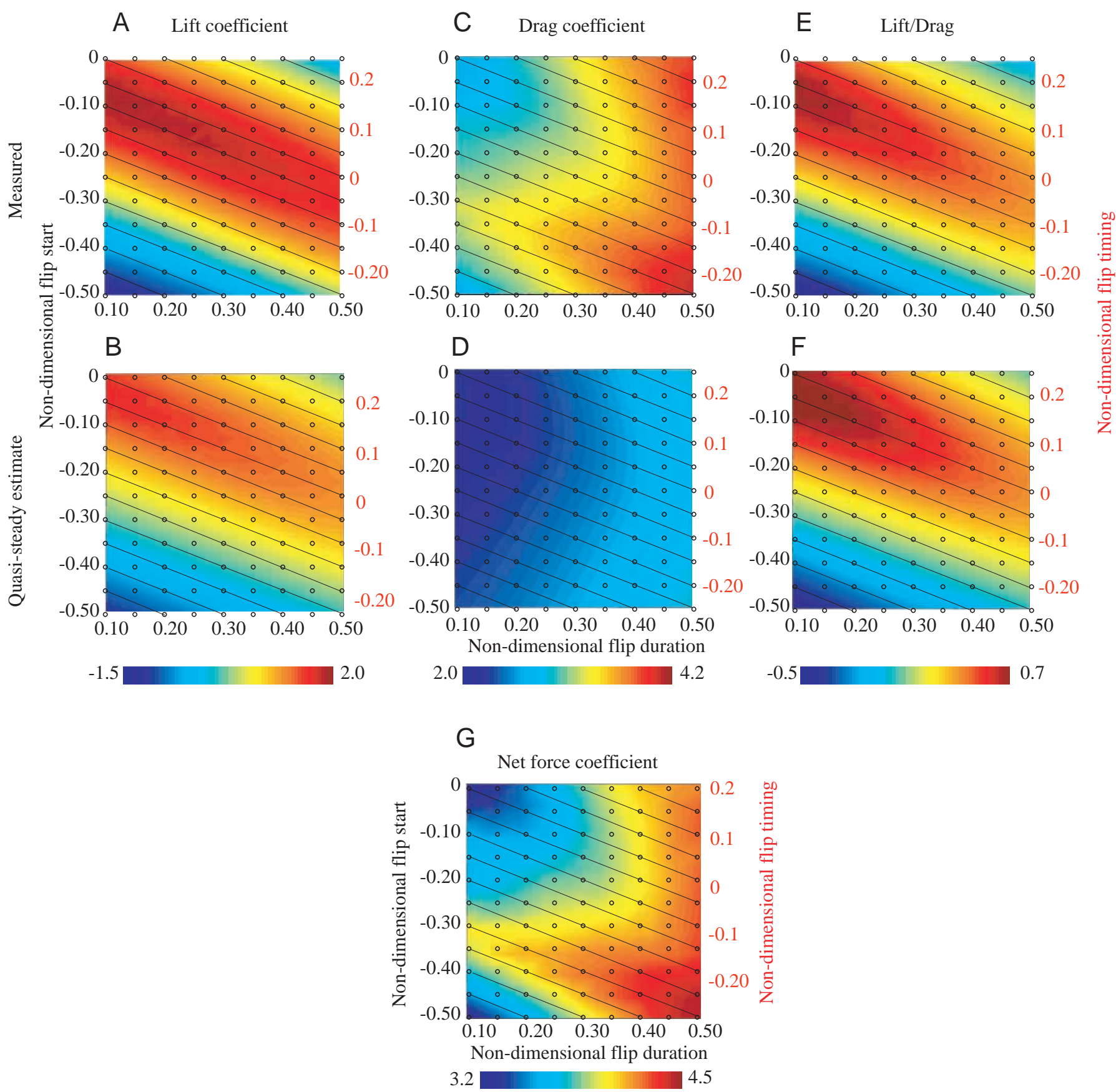

Fig. 7. Parameter maps of force coefficients as a function of flip start and flip duration. Each map was generated from a $9 \times 11$ array of kinematic patterns. For all experiments, the stroke amplitude $\Phi$ and angle of attack $\alpha$ were held constant $\left(\Phi=180^{\circ}, \alpha=45^{\circ}\right)$, while flip duration and flip start were systematically varied. The data and interpolated values are plotted as in Fig. 4 and Fig. 5. Isolines of flip timing (given by equation 2) are indicated by the diagonal lines in each panel and correspond to the red labels on the right axis. The top panels (A,C,E) show the measured force coefficients, and the bottom panels $(\mathrm{B}, \mathrm{D}, \mathrm{F})$ show values for the quasi-steady-state estimated coefficients. Axis labels given in A apply to all panels. (A,B) Measured and quasi-steady values for stroke-averaged mean lift coefficient. The pseudocolor scale for both panels is shown below the parameter map in B. (C,D) Measured and quasi-steady values for stroke-averaged mean drag coefficient. Note the large discrepancy between the estimated and measured values. (E,F) Measured and quasi-steady values for stroke-averaged mean lift-to-drag ratio. (G). Measured values for stroke-averaged mean net force coefficient. Note the strong similarity to the drag coefficient map.

stronger if the wing moves downwards, towards the descending vorticity of the previous stroke, than if it moves upwards, away from the wake. In all cases, the significance of radial forces increases with increasing deviation. As expected, like the lift and drag forces, the time course of radial forces is also dependent on the shape of the wing trajectory.

Because of the mirror symmetry in upstroke and downstroke kinematics for 'oval' kinematics (Fig. 8A,B), the radial forces 
Fig. 8. Sample instantaneous forces for various combinations of stroke deviation and tip trajectory. All other kinematic variables were constant (amplitude $\Phi=180^{\circ}$, angle of attack $\alpha=45^{\circ}$, flip duration $\Delta \tau=0.5$, flip timing $\tau_{\mathrm{f}}=-0.05$ ). The format for each panel is that described for Fig. 3 and Fig. 6, except for an additional panel showing the instantaneous radial forces (green). (A) Forces generated with a large oval deviation in which the downstroke starts with upward motion and the upstroke starts with downward motion $D_{\mathrm{dev}}=+25^{\circ}$, $U_{\mathrm{dev}}=-25^{\circ}$, where $D_{\mathrm{dev}}$ is equal to the maximum angle of downstroke deviation and $U_{\mathrm{dev}}$ indicates maximum angle of upstroke deviation. Both lift and drag transients are higher at the start of the upstroke when the wing travels downwards than at the start of the downstroke. The absolute average radial forces are also correspondingly large. (B) Reversed condition compared with A, the downstroke starts with downward motion and the upstroke starts with upward motion $\left(D_{\mathrm{dev}}=-25^{\circ}, U_{\mathrm{dev}}=+25^{\circ}\right)$. Lift and drag transients are now much larger at the start of the downstroke. (C) Forces generated with a figure-ofeight deviation in which both strokes start with upward motion $\left(U_{\mathrm{dev}}=D_{\mathrm{dev}}=+25^{\circ}\right)$. Both lift and drag are low at the start of each stroke, but reach elevated values at midstroke. (D) Reversed condition compared with $\mathrm{C}$, both strokes start with downward motion $\left(U_{\mathrm{dev}}=D_{\mathrm{dev}}=-25^{\circ}\right)$. Both strokes now start with large transients in both lift and drag. (E) Forces generated by comparable kinematic pattern to those in A-D but with no stroke deviation.
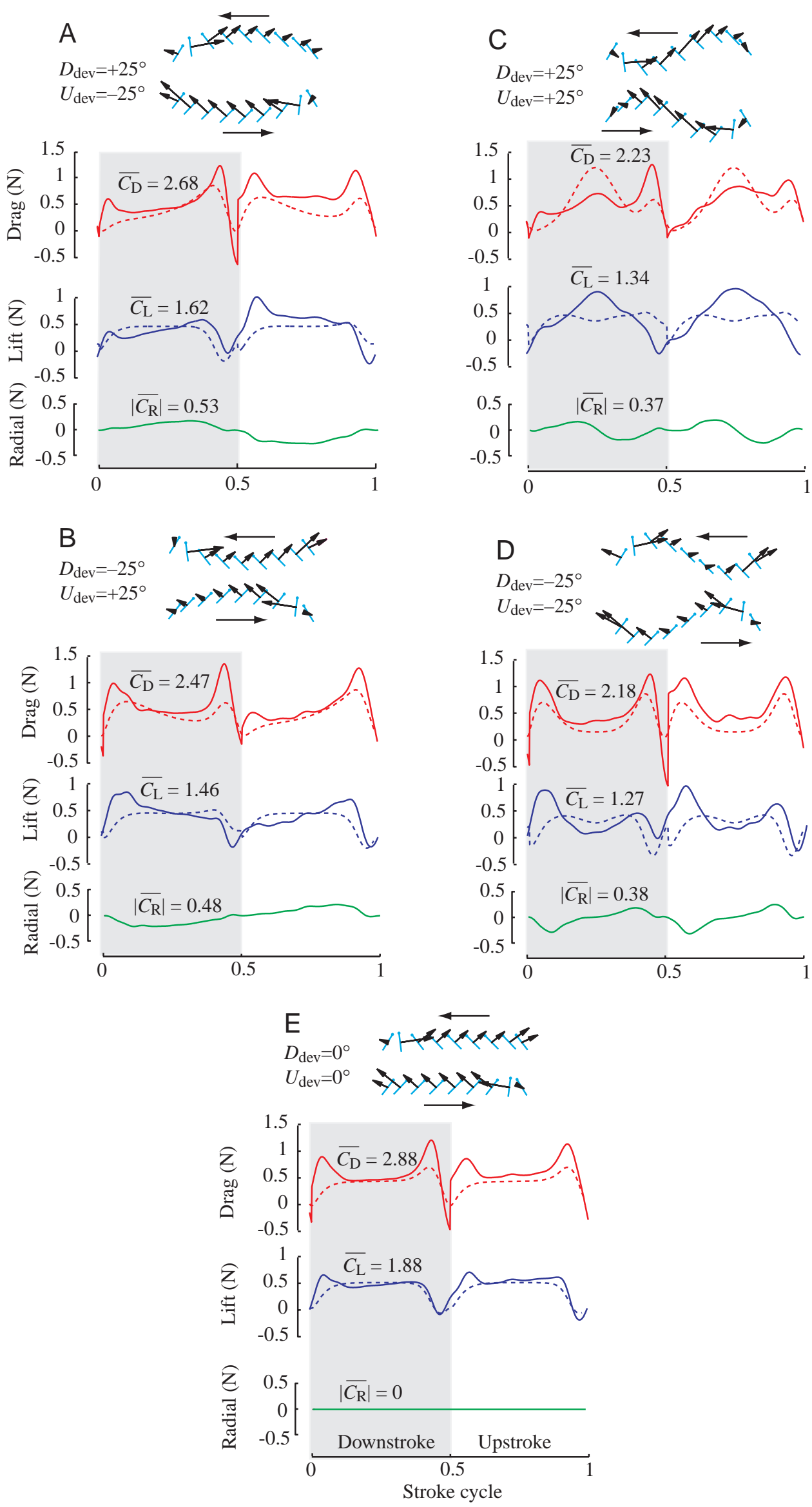
Fig. 9. Effects of stroke deviation on mean force coefficients. (A) Cartoon illustrating the kinematic patterns used to study stroke deviation. Throughout the figure, blue data points refer to oval kinematic patterns, and red data points refer to figure-of-eight patterns. Measured and quasisteady predicted values are given by filled and open circles, respectively. (B) The magnitude of the mean net aerodynamic force coefficient is only mildly influenced by stroke deviation. (C) Measured mean lift coefficient decreases with stroke deviation. For the figure-of-eight patterns, the drop in performance with increasing deviation is greater for strokes that begin with upward motion. (D) Drag coefficient decreases with stroke deviation. Note the large discrepancy between measured values of mean drag and quasi-steady predictions. (E) Ratio of lift-to-drag and radialto-drag forces as a function of stroke deviation. Radial-to-drag forces for oval kinematic patterns are represented by filled black circles and for figure-of-eight patterns by open black circles. Because deviation affects lift and drag almost equally, the influence on their ratio is quite small. The radial forces are dependent on the sine of stroke deviation angle. Because of the linearity of sine functions for small angles, the values of radial-to-drag force increase linearly with increasing absolute deviation.
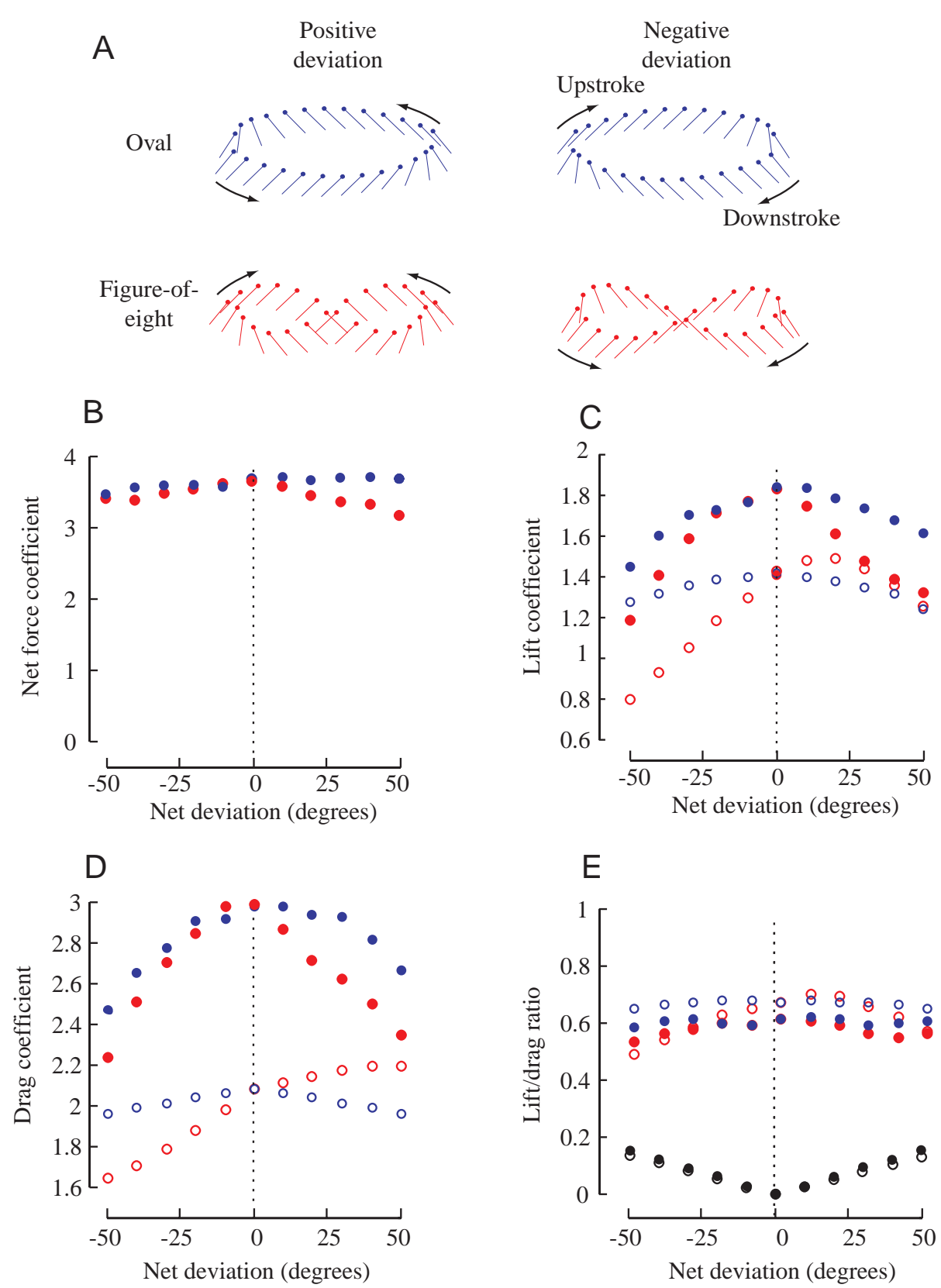

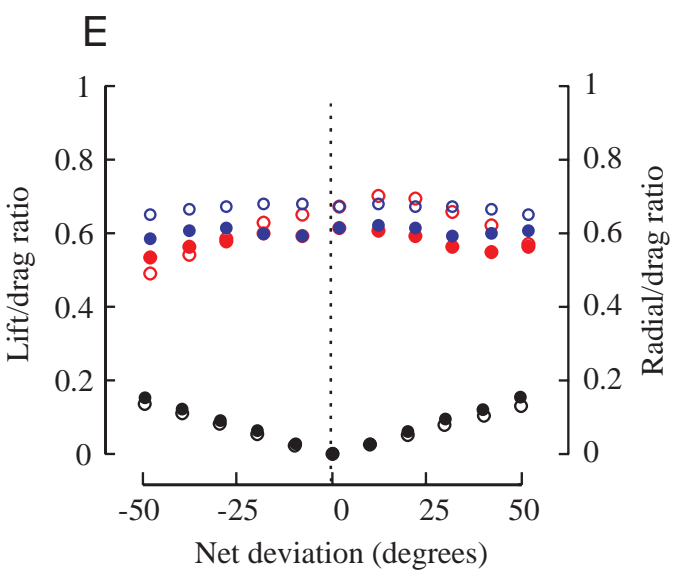

during the upstroke are equal and opposite to the radial forces during the downstroke. In the 'figure-of-eight' kinematics, however, the upstroke and downstroke are identical and, hence, the radial forces during the upstroke are identical to the radial forces during the downstroke.

Fig. 9 summarizes the effects of stroke deviation on the time-averaged measured forces and quasi-steady predictions. While the influence of stroke deviation on the time course of the aerodynamic forces is large, its impact on the mean force coefficients is surprisingly small. This indicates that the differences in the dynamics of force production noted in Fig. 8 tend to average out over the stroke. For both the 'oval' and 'figure-of-eight' deviation trajectories (Fig. 9A), the mean lift and drag coefficients decreased with increasing absolute deviation (Fig. 9C,D). The changes in average performance for the 'oval' deviation pattern should be symmetrical around zero deviation, since oval patterns with positive and negative deviations are mirror images of one another. Thus, the downstroke in Fig. 8A should resemble the upstroke in Fig. 8B, and the upstroke in Fig. 8A should resemble the downstroke in Fig. 8B. The asymmetry in these measurements results from the mechanical play in the gear mechanism of the robot. However, the asymmetry in the performance of the 'figure-of-eight' patterns around zero deviation represents, in part, a real aerodynamic effect (Fig. 9B,C,D). In this case, a positive deviation will result in a downward motion at the start of both the upstroke and downstroke, whereas a negative deviation indicates upward motion at the beginning of both strokes. Downward deviation should enhance wake capture, as described above. Values of $\overline{C_{\mathrm{L}}}$ and $\overline{C_{\mathrm{D}}}$ fall off faster with 
increasing positive deviation close to zero deviation. However, at large deviations, the coefficients for negative deviations are lower than the coefficients for positive deviations (Fig. 9C,D). There is little effect on the $\overline{C_{\mathrm{L}}} / \overline{\mathrm{C}_{\mathrm{D}}}$ ratio because the influence of stroke deviation is nearly identical for both lift and drag. In contrast, because of the linear nature of sine functions at low angles, $\overline{C_{\mathrm{L}}} / \overline{C_{\mathrm{D}}}$ ratios appear linear with the small range of stroke deviation in our experiments (Fig. 9E).

As with the earlier experiments, the measured values of both $\overline{C_{\mathrm{L}}}$ and $\overline{C_{\mathrm{D}}}$ are higher than quasi-steady translational estimates due to unsteady effects (Fig. 9C,D). Lift and drag are almost equally underestimated, which explains why the predicted liftto-drag ratio (Fig. 9E) is only slightly higher than the measured ratio. For oval trajectories, the quasi-steady predictions for the mean force coefficients behave as scaled-down versions of the measured traces. For example, both quasi-steady and measured mean force coefficients are maximal at zero stroke deviation and decrease for increasing absolute deviation (Fig. 9C,D). However, for figure-of-eight trajectories, the quasi-steady translational model is much less accurate in predicting the changes in the mean force coefficients with stroke deviation. For example, the quasi-steady model predicts that mean lift should exhibit a local maximum at a stroke deviation of $+20^{\circ}$, whereas the measured maximum occurs at $0^{\circ}$ deviation. Similarly, the estimated drag increases monotonically with increasing positive deviation, whereas the measured drag is maximal at $0^{\circ}$ deviation.

\section{Ratio of mean lift to mean profile power}

Within the range of Reynolds numbers relevant for fruit flies, the total mechanical power required to flap the wings is dominated by profile power, $\overline{P_{\text {pro }}}$, the cost to overcome drag on the flapping wings (Lehmann and Dickinson, 1997). Fig. $10 \mathrm{~A}-\mathrm{C}$ shows how $\overline{P_{\text {pro }}}$, estimated using equation 6 , varies with changes in the kinematics parameters. Fig. 10D-F shows the corresponding ratios of mean lift to mean profile power for the same kinematics. The $\overline{P_{\text {pro }}}$ estimates vary extensively, even within subregions of the parameter maps in which the values of $\overline{C_{\mathrm{L}}}$ are high enough to support flight. This result suggests that it may be difficult to estimate mechanical power in free or tethered flight solely on the basis of measures of stroke amplitude. In particular, $\overline{P_{\text {pro }}}$ varies extensively with the timing of wing rotation and the angle of attack, parameters that are revealed only by extensive three-dimensional reconstructions of wing kinematics.

\section{Discussion}

We have used a dynamically scaled model wing to measure both instantaneous and stroke-averaged lift and drag forces for a wide variety of behaviorally relevant kinematic patterns on a flapping wing. The results underscore the importance of three unsteady mechanisms in flapping flight: delayed stall, rotational circulation and wake capture. The processes are important not simply because they help to explain the lift required to keep an animal aloft but also because their sensitivity to subtle changes in stroke kinematics may help to explain the extreme maneuverability of many insects. By systematically measuring flight forces within a comprehensive kinematic space, we have begun to untangle the complex interactions among these force-generating mechanisms.

\section{Comparison between the mechanical model and real flies}

The maximum mean unsteady lift coefficients in this study are in excellent agreement with measurements on tethered Drosophila spp., which generated elevated flight force in response to optomotor stimuli. Peak $\overline{C_{\mathrm{L}}}$ values in tethered flight were 1.9 (Lehmann and Dickinson, 1998), which is precisely the same maximum value measured on the model wing. Tethered flies generating just enough force to support body weight produce a $\overline{C_{\mathrm{L}}}$ value of 1.6. The tethered flight estimates relied on the assumption that both up- and downstrokes generated lift, which appears to be correct given the time course of forces generated when Drosophila spp. kinematics are played through the model (Dickinson et al., 1999). Tethered Drosophila spp. flap with morphologically maximum stroke amplitude when producing peak lift (Lehmann and Dickinson, 1998), which is consistent with the present results. Unfortunately, apart from stroke amplitude, we do not have adequate knowledge of other kinematic parameters during the peak performance of real flies to compare them with the values that maximized lift on the model.

As seen in Fig. 5B, the maximum quasi-steady translational estimate of the mean lift coefficient, $\overline{C_{\mathrm{L}, \mathrm{t}}}$, was 1.6 . Given that this value is sufficient to explain the forces required for a fruit fly to hover (Lehmann and Dickinson, 1998), it is tempting to claim that the quasi-steady estimates are sufficient to explain insect flight (Jensen, 1956). However, a simple comparison of time-averaged lift coefficients is not a robust test of the quasisteady model. While the mean values might be similar, the time histories of measured forces and quasi-steady translational estimates differ greatly (Fig. 3, Fig. 6, Fig. 8). Further, the precise time history of lift and drag is critical for calculating force moments and, thus, essential to considerations of stability and maneuverability. In addition, since many insects can fly while supporting forces nearly twice their body weight (Marden, 1987), any model must explain not only the forces required to hover, but also those required for maximal lift.

The inadequacies of the translational quasi-steady model are even more apparent when considering drag. In all experiments, we observed that the measured drag coefficients were greater in magnitude than those estimated from measured translational force coefficients. Also, the range of drag coefficients measured here $\left(C_{\mathrm{D}}=0-6.5\right)$ is substantially higher than previously reported measurements from both real and model Drosophila virilis wings $\left(C_{\mathrm{D}}=0.2-1\right.$; Vogel, 1967). The previous experimental values were based on steady-state measurements and thus excluded the contribution of rotational circulation and wake capture as well as added mass. However, even the range of steady-state coefficients in the previous study $\left(0<C_{\mathrm{L}}<1,0<C_{\mathrm{D}}<1\right.$; Vogel, 1967) is substantially lower than the quasi-steady estimates in this study $\left(0<C_{\mathrm{L}, \mathrm{t}}<1.91\right.$; 

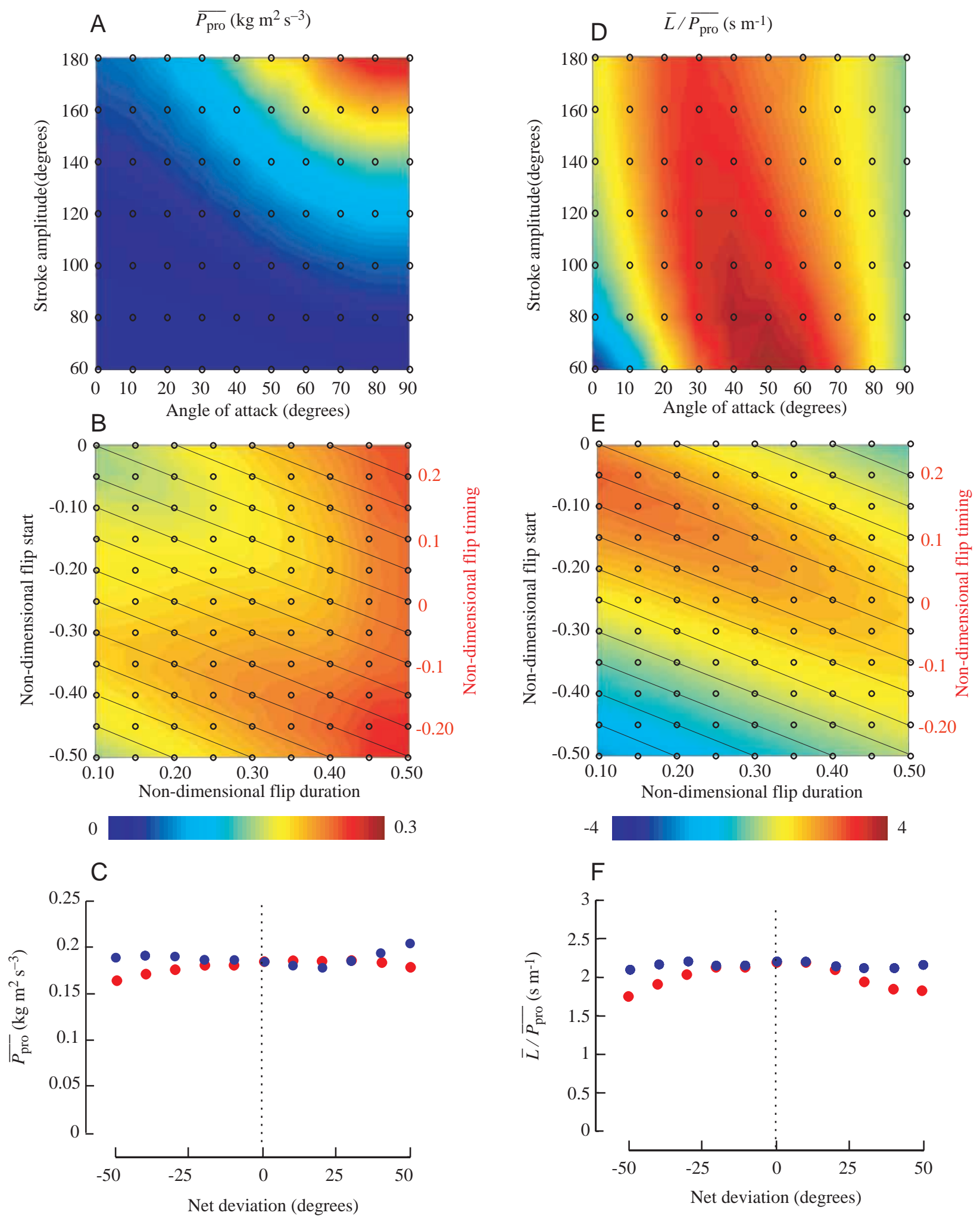

Fig. 10

$\left.0.37<C_{\mathrm{D}, \mathrm{t}}<3.47\right)$. At present, the reason for this discrepancy is not clear.

The relative importance of unsteady mechanisms

The relative contributions of delayed stall, rotational circulation and wake capture to total force production vary with the precise kinematics of the stroke. In general, the importance of delayed stall increases with stroke amplitude because the wing can integrate the influence of the leading edge vortex over a greater distance. In contrast, rotational 
Fig. 10. The effects of wing kinematics on profile power $\overline{P_{\text {pro }}}$ and the lift-to-power ratio $\bar{L} / \overline{P_{\text {pro }}}$. Procedures for plotting data are as described in Fig. 4, Fig. 7 and Fig. 9. The pseudocolor scale for A and $\mathrm{B}$ is shown below $\mathrm{B}$, and the scale for $\mathrm{D}$ and $\mathrm{E}$ is shown below E. (A) Profile power as a function of stroke amplitude and angle of attack. (B) Profile power as a function of flip start and flip duration. Flip timing is shown on the right axis. Profile power varies by a factor of 2 within the parameter space, indicating that flip kinematics are important determinants of flight cost. (C) Profile power as a function of stroke deviation for oval (blue) and figure-of-eight (red) patterns. Stroke deviation has only a minor effect on profile power. (D) The ratio of mean lift to mean profile power, $\bar{L} / \overline{P_{\text {pro }}}$, as a function of stroke amplitude and angle of attack. Like the mean lift coefficient (Fig. 5A), there is a single angle of attack that maximizes $\bar{L} / \overline{P_{\text {pro }}}$ for each value of stroke amplitude. (E) The ratio of mean lift to mean profile power as a function of flip start, flip duration and flip timing. (F) The ratio of mean lift to mean profile power as a function of stroke deviation for oval (blue) and figure-of-eight (red) deviation.

effects become more important with lower stroke amplitude. For kinematic patterns mimicking those of hoverflies with a $60^{\circ}$ stroke amplitude, rotational effects account for more than half the total force (Dickinson et al., 1999). Because of these differences, quasi-steady translational estimates should more closely resemble the measured values for kinematic patterns with large stroke amplitudes and deviate from measured values for those with lower stroke amplitudes. These predictions are borne out by the force traces shown in Fig. 3 and the maps of $\overline{C_{\mathrm{L}}}$ in Fig. 5A,B. The quasi-steady translational model predicts maximum lift at an angle of attack of $45^{\circ}$. While this is close to the maximum value for the measured forces at high stroke amplitude, the measured lift maximum is shifted to higher angles of attack $\left(60^{\circ}\right)$ at low stroke amplitude.

There are two possible explanations for this shift. First, rotational circulation might make a greater contribution to mean lift at low stroke amplitude. However, this is unlikely to be true for symmetrical flips, for which rotational circulation enhances lift only before stroke reversal, but attenuates it after stroke reversal (Dickinson et al., 1999). Also, at stroke reversal, the wing rotates less for a $60^{\circ}$ angle of attack than it does for a $45^{\circ}$ angle of attack. Thus, the proportional contribution due to rotational lift is further minimized. Second, the shift in the lift maximum may reflect an increasing importance of wake capture at low stroke amplitude. The large contribution of wake capture can be easily seen in the forces generated by hoverfly-like patterns (Dickinson et al., 1999) as well as in the drag traces in Fig. 3. The increasing importance of wake capture also explains the changes in drag coefficients at low stroke amplitude. While the translational quasi-steady model predicts that drag should be independent of amplitude (Fig. 5D), the measured mean drag coefficient clearly increases at smaller stroke amplitudes.

\section{Separating the effects of wing rotation from wake capture}

Since rotational circulation, wake capture and added mass usually occur together during stroke reversal, it is often difficult to separate these effects. After estimating the effect of added mass on force traces before and after subtraction (Fig. 2), we concluded that, at these Reynolds numbers, the magnitude of added mass is small compared with rotational circulation or wake capture. To isolate these two mechanisms, it is helpful to focus on kinematic patterns in which either the entire flip occurs prior to stroke reversal (Fig. 6B,E,F) or the wing does not flip at all (Fig. 3A,B). In the case of an advanced flip, the force peak that exceeds the translational estimate prior to stroke reversal may be attributed to rotational circulation, while the force peak after stroke reversal is due to wake capture. In the case of no flip, the large drag peak at the start of each stroke is due to wake capture, in which vorticity shed from the previous stroke elevates force by inducing an increase in flow velocity towards the wing (Dickinson, 1994; Dickinson et al., 1999). Because of the squared dependence of forces on relative velocity, even small changes in flow can cause a large elevation in force.

The influence of wake capture should be reduced when the wing translates at a $0^{\circ}$ angle of attack during the previous stroke. Under these circumstances, the wing sheds less vorticity at the end of each stroke, since it did not create a leading edge vortex during translation. There is some vorticity present, however, due to rotational circulation at the end of the stroke. Nevertheless, in these cases (Fig. 3G,H), wake capture is reduced, which partially isolates the influence of rotational circulation. The results indicate that a wing rotating early enhances lift, but that if the rotation continues after stroke reversal the force dips below the quasi-steady-state prediction because, after changing direction, rotational circulation will act to counter the circulation produced by translation.

By systematically varying the kinematic parameters, we determined that lift was optimized at a $45^{\circ}$ angle of attack, $180^{\circ}$ stroke amplitude, $10 \%$ flip duration and approximately $-5 \%$ flip advance. Even subtle deviations from the optimal combination drastically decreased lift. A $5 \%$ delay in flip timing relative to the optimal conditions (Fig. $6 \mathrm{H}$ ) is less effective in generating rotational lift and decreases the average coefficient by as much as $25 \%$. Small decreases in flip duration cause comparable changes. These results suggest that the control of flip timing and duration provides an easy and powerful way of modulating the forces on two wings, as is required to alter force moments during steering. Tethered flies advance wing rotation on the outside of a turn in response to a turning visual stimulus (Dickinson et al., 1993). Since early rotation enhances whereas delayed rotation attenuates lift, early wing rotation on the outside of a turn should produce a greater force on that side, thus creating an appropriate turning moment in the opposite direction. While flip advance is usually coupled with an increase in stroke amplitude, flies do possess the ability to decouple the modulation of rotation and amplitude (Dickinson et al., 1993). A more thorough analysis of steering must consider the influence of wing kinematics on force moments, which will be the topic of a separate paper (S. P. Sane and M. H. Dickinson, in preparation)

In nearly all the experiments presented here, the kinematics of the upstroke and downstroke were identical. Under these 
conditions, the contributions of the upstroke and downstroke to forces are equal, and the net force vector is oriented normal to the mean stroke plane. However, symmetrical wing strokes are probably more the exception in actual insects than the rule. Thus, further experiments must focus on asymmetric strokes that can cause the net force vector to be tilted relative to the stroke plane. Adjusting the symmetry of the two strokes is undoubtedly critical for the regulation of force moments in flight control.

\section{The influence of stroke deviation}

The wings of insects do not always beat back and forth within a flat stroke plane, but may exhibit large and complex deviations. Within studies of flies, the true form and function of these deviations have been subject to much debate (for a review, see Dickinson and Tu, 1997). While the existence of oval and figure-of-eight trajectories was often attributed to differences in experimental procedure (tethered versus free flight, thoracic versus abdominal tether, still air versus moving air), studies of neuromuscular control indicate that flies have the ability actively to modulate stroke deviation by altering the activity of steering muscles (Tu and Dickinson, 1994; Lehmann and Götz, 1996). The aerodynamic utility of these changes, however, is still not known.

We can divide the effects of stroke deviation on aerodynamic mechanisms into three categories: the introduction of radial force components, alteration of aerodynamic angle of attack and the efficacy of wake capture. The presence of one or more of these effects can substantially alter the dynamics of force generation even when the timeaveraged net force produced by the wing remains largely unaltered (filled circles, Fig. 9B). For three-dimensional flapping, the main influence of stroke deviation on flight forces is the presence of radial force components acting orthogonal to lift and drag (Fig. 8A-E). This force component, although ignored in much of the insect flight literature, is of considerable importance during large stroke deviations. Although the magnitude of the average net force vector remains nearly constant with changes in deviation, both lift and drag coefficients decrease with increasing positive and negative stroke deviations, while the radial component increases. In this way, deviation offers a versatile means of manipulating forces through simple geometric alterations in stroke trajectory.

Stroke deviation also influences force production by altering the angle of attack with respect to the wing path as well as its instantaneous velocity. Upward motion of the wing at the start of the stroke results in a decrease in the angle of attack, while downward motion causes an increase in the angle of attack. The converse is true at the end of the stroke. In addition, the velocity of the wing is higher, because the wing must cover a greater distance when it follows a curved trajectory for a given stroke frequency. This effect is clear in the quasi-steady prediction for the oval wingbeat patterns (Fig. 8A,B). The predicted forces are higher at the start of strokes beginning with downward motion and at the end of strokes that finish with downward motion. However, a comparison of measured and predicted force traces indicates that the wing generates large force transients at the start of strokes beginning with downward motion that cannot be explained by the changes in aerodynamic angle of attack and wing velocity, which are incorporated in the quasi-steady model (Fig. 8B,D). The most likely explanation for these large peaks is that wake capture is enhanced by downward motion and attenuated by upward motion at the start of the stroke. By moving downwards in the direction of the descending wake, the wing may intercept a stronger flow field than if it moved upwards. However, a rigorous test of this hypothesis will require a detailed visualization of the velocity distribution during stroke reversal.

The interaction between stroke deviation and wake capture is more extreme in the case of figure-of-eight patterns, in which the two half-strokes are mirror images of one another and can thus both begin with downward motion. The quasi-steady model predicts that mean lift should be maximal with a $+20^{\circ}$ deviation, which means that the wing begins each stroke by moving upwards. The enhanced performance of upward strokes is due to the fact that the angle of attack is high in the middle of the stroke when the wing velocity is greatest. The measured forces do not, however, exhibit this enhanced performance with small positive deviations. On the contrary, the net performance drops off more quickly with positive deviation (upward motion at start of strokes) than with negative deviation (downward motion at start of stroke). This result is again consistent with a more effective wake capture due to downward motion at the start of translation.

In spite of the large differences in instantaneous forces, the magnitudes of time-averaged forces in all cases are similar for the oval and figure-of-eight trajectories (Fig. 9). For oval trajectories, the measured forces behave as predicted, with maximum lift and drag coefficients occurring at zero deviation and decreasing for higher absolute deviation. In contrast, the figure-of-eight trajectories behave quite differently from the corresponding quasi-steady estimates, varying in both magnitude and behavior. For quasi-steady estimates, the lift maximum occurs at a $20^{\circ}$ positive deviation, whereas the drag maximum increases monotonically with increasing positive deviations (open red circles in Fig. 9C,D respectively). In contrast, the measured values in both cases are maximal at zero deviation (filled blue and red circles in Fig. 9C,D) and then decrease for higher absolute deviations.

\section{Drag and efficiency}

The values of mean profile drag $\overline{C_{\mathrm{D} \text {,pro }}}$ obtained in the current study are much higher than values in the literature based either on steady-state measurements of wing profiles (Vogel, 1967) or calculations based on Reynolds number (Ellington, 1984d; Lehmann and Dickinson, 1997; Wakeling and Ellington, 1997). In particular, the equation:

$$
\overline{C_{\mathrm{D}, \text { pro }}}=7 / \sqrt{\operatorname{Re}} \text {, }
$$

which represents the rough average of the Reynolds-numberdependence of a flat plate parallel and perpendicular to flow (Ellington, 1984c), may grossly underestimate the profile drag 
on a flapping wing. In the current study, this equation predicts a $\overline{C_{\mathrm{D}, p r o}}$ value of approximately 0.7 for large stroke amplitudes, which is less than $25 \%$ of the measured values for kinematic patterns capable of sustaining flight $\left(\overline{C_{\mathrm{D}, \mathrm{t}}} \approx 3\right)$. One obvious reason for this large discrepancy is that the process of wing rotation generates large instantaneous values of drag which, even though lasting only a small portion of the stroke, substantially increase the mean values. However, even the drag contributed by delayed stall during translation $(\approx 2)$ is higher than that predicted by equation 7 . These large values for the mean drag coefficient have several important implications for flight energetics and muscle physiology. First, profile power, which scales linearly with the mean drag coefficient, may be several times greater than predicted in estimates using equation 7 . Thus, under many conditions, profile power, and not induced power, may represent the dominant component in aerodynamic power. Further, estimates of total mechanical power based solely on induced power are likely grossly to underestimate flight costs. The elevated values of drag also call into question previous attempts to estimate elastic storage, which depend upon inertial power costs being high relative to aerodynamic costs (Dickinson and Lighton, 1995). If the ratio of aerodynamic cost to inertial cost is high, the energetic benefits of elastic storage are greatly reduced. Second, because previous estimates of mechanical power may be several times too low, calculations of muscle efficiency, based on the ratio of mechanical power to total metabolic power, may be substantially lower than actual values. Such estimates for the muscle efficiency of asynchronous flight muscle range from 8 to $12 \%$. When corrected for the underestimate of drag, we find that previous estimates of muscle efficiency presented for fruit flies (Lehmann and Dickinson, 1997) should be adjusted to 20-25\%. If correct, these adjusted values help to solve a perplexing problem in insect flight physiology. Because $\mathrm{Ca}^{2+}$ cycling represents a sizeable fraction of the energetic cost in cyclic contractions, stretch-activated muscle should accrue an energetic savings and concomitant increase in mechanical efficiency. This prediction was not supported, however, by the previous estimates of efficiency. After correction with more accurate values of drag, the estimates of mechanical efficiency in asynchronous muscle are now consistent with the reduced cost of $\mathrm{Ca}^{2+}$ cycling.

In addition to indicating that the mean level of drag may be higher than previously expected, the results also show that the value of drag is quite sensitive to subtle changes in wing kinematics (Fig. 5C, Fig. 7C, Fig. 9D). Mapping an estimate of profile power indicates that this sensitivity of the drag coefficient to wing kinematics translates into a sensitivity of flight cost as well (Fig. 10A,B,C). This result again has consequences for estimates of flight costs and mechanical efficiency from free-flight kinematics based solely on measures of stroke frequency and amplitude (Ellington, 1984d). In particular, for a given stroke amplitude, profile power varies depending upon the timing and duration of wing rotation (Fig. 10B). Thus, without knowledge of the precise stroke kinematics, estimates of free-flight energetics must be viewed with caution.

\section{Concluding remarks}

The data presented in this paper represent a comprehensive analysis of the effects that wing kinematics have on the generation of aerodynamic forces in flapping flight. The results reveal a complex system in which subtle alterations in stroke kinematics can have quite large effects on force production. In future studies, it will be an important challenge to determine how real insects use this sensitively to adjust the balance of forces and force moments as they steer and maneuver through their environment.

\section{List of symbols}

$\begin{array}{ll}A R & \text { aspect ratio } \\ \bar{c} & \text { mean chord length } \\ \hat{c}(\hat{r}) & \text { non-dimensional chord length } \\ \overline{C_{\mathrm{D}, \text { pro }}} & \text { mean profile drag coefficient } \\ \overline{C_{\mathrm{D}}} & \text { mean drag coefficient } \\ C_{\mathrm{D}, \mathrm{t}} & \text { translational drag coefficient } \\ \overline{C_{\mathrm{F}}} & \text { mean force coefficient } \\ \overline{C_{\mathrm{L}}} & \text { mean lift coefficient } \\ C_{\mathrm{L}, \mathrm{t}} & \text { translational lift coefficient } \\ \overline{C_{\mathrm{N}}} & \text { mean total aerodynamic force coefficient } \\ \overline{C_{\mathrm{R}}} & \text { mean radial coefficient } \\ \overline{\mathrm{d} \hat{\phi} / \mathrm{d} \hat{t}} & \text { mean non-dimensional angular velocity of the wing } \\ D & \text { drag } \\ D_{\mathrm{dev}} & \text { maximum angle of downstroke deviation } \\ D(t) & \text { instantaneous drag } \\ \bar{F} & \text { force coefficient averaged over a stroke } \\ F_{\mathrm{a}, \mathrm{N}} & \text { added mass inertia normal to the wing surface } \\ \overline{F_{\mathrm{N}}} & \text { mean total aerodynamic force } \\ L & \text { lift } \\ n & \text { wingbeat frequency } \\ \overline{P_{\mathrm{pro}}} & \text { mean profile power } \\ R & \text { wing length } \\ R e & \text { Reynolds number } \\ \hat{r} & \text { non-dimensional radial position along wing length } \\ \hat{r}_{2}{ }^{2}(S) & \text { non-dimensional second moment of wing area } \\ S & \text { surface area of a wing pair } \\ t & \text { time } \\ T & \text { stroke period } \\ U_{\mathrm{dev}} & \text { maximum angle of upstroke deviation } \\ v_{\text {wing }}(t) & \text { instantaneous wing velocity } \\ \alpha & \text { angle of attack } \\ \alpha(t) & \text { instantaneous angle of attack } \\ \Delta \tau & \text { flip duration } \\ \Phi & \text { total stroke amplitude } \\ \phi(t) & \text { instantaneous stroke position } \\ \nu & \text { kinematic viscosity } \\ \theta(t) & \text { instantaneous stroke deviation } \\ \Theta & \text { maximum stroke deviation } \\ \rho & \text { density of fluid } \\ \tau_{\mathrm{f}} & \text { flip timing } \\ \tau_{0} & \text { flip start } \\ & \end{array}$

This work was supported by grants from the NSF (IBN- 
9723424), Defense Advanced Research Projects Agency and the Office of Naval Research (FDN00014-99-1-0892). We wish to thank Fritz Lehmann, Claire Balint, Namrata Gundiah, Jim Birch and two anonymous reviewers for helpful comments.

\section{References}

Arbas, E. (1986). Control of hindlimb posture by wind-sensitive hairs and antennae during locust flight. J. Comp. Physiol. A 159, 849-857.

Bennett, L. (1970). Insect flight: lift and the rate of change of incidence. Science 167, 177-179.

Bennett, L. (1977). Clap and fling aerodynamics - an experimental evaluation. J. Exp. Biol. 69, 261-272.

Cloupeau, M., Devillers, J. F. and Devezeaux, D. (1979). Direct measurements of instantaneous lift in desert locust; comparison with Jensen's experiments on detached wings. J. Exp. Biol. 80, 1-15.

Daniel, T. L. (1984). Unsteady aspects of aquatic locomotion. Am. Zool. 24, 121-134.

David, C. T. (1978). The relationship between body angle and flight speed in free flying Drosophila. Physiol. Ent. 3, 191-195.

Dickinson, M. H. (1994). The effects of wing rotation on unsteady aerodynamic performance at low Reynolds numbers. J. Exp. Biol. 192, 179-206.

Dickinson, M. H. and Götz, K. G. (1993). Unsteady aerodynamic performance of model wings at low Reynolds numbers. J. Exp. Biol. 174, 45-64.

Dickinson, M. H. and Götz, K. G. (1996). The wake dynamics and flight forces of the fruit fly Drosophila melanogaster. J. Exp. Biol. 199, 2085-2104.

Dickinson, M. H., Lehmann, F.-O. and Götz, K. G. (1993). The active control of wing rotation by Drosophila. J. Exp. Biol. 182, 173-189.

Dickinson, M. H., Lehmann, F.-O. and Sane, S. P. (1999). Wing rotation and the aerodynamic basis of insect flight. Science 284, 1954-1960.

Dickinson, M. H. and Lighton, J. R. B. (1995). Muscle efficiency and elastic storage in the flight motor of Drosophila. Science 128, 87-89.

Dickinson, M. and Tu, M. (1997). The function of dipteran flight muscle. Comp. Biochem. Physiol. 116A, 223-238.

Ellington, C. P. (1984a). The aerodynamics of hovering insect flight. II. Morphological parameters. Phil. Trans. R. Soc. Lond. B 305, 17-40.

Ellington, C. P. (1984b). The aerodynamics of hovering insect flight. III. Kinematics. Phil. Trans. R. Soc. Lond. B 305, 41-78.

Ellington, C. P. (1984c). The aerodynamics of hovering insect flight. IV. Aerodynamic mechanisms. Phil. Trans. R. Soc. Lond. B 305, 79-113.

Ellington, C. P. (1984d). The aerodynamics of hovering insect flight. VI. Lift and power requirements. Phil. Trans. R. Soc. Lond. B 305, 145-181.

Ellington, C., Vandenberg, C., Willmott, A. and Thomas, A. (1996). Leading-edge vortices in insect flight. Nature 384, 626-630.

Ennos, A. R. (1989). The kinematics and aerodynamics of the free flight of some Diptera. J. Exp. Biol. 142, 49-85.

Götz, K. G., Hengstenberg, B. and Biesinger, R. (1979). Optomotor control of wing beat and body posture in Drosophila. Biol. Cybernetics 35, $101-112$.
Jensen, M. (1956). Biology and physics of locust flight. III. The aerodynamics of locust flight. Phil. Trans. R. Soc. Lond. B 239, 511-552.

Lehmann, F.-O. and Dickinson, M. H. (1997). The changes in power requirements and muscle efficiency during elevated force production in the fruit fly Drosophila melanogaster. J. Exp. Biol. 200, 1133-1143.

Lehmann, F. and Dickinson, M. (1998). The control of wing kinematics and flight forces in fruit flies (Drosophila spp.). J. Exp. Biol. 201, 385-401.

Lehmann, F. and Götz, K. (1996). Activation phase ensures kinematic efficacy in flight-steering muscles of Drosophila melanogaster. J. Comp. Physiol. A 179, 311-322.

Liu, H., Ellington, C. P., Kawachi, K., VandenBerg, C. and Willmott, A. P. (1998). A computational fluid dynamic study of hawkmoth hovering. $J$. Exp. Biol. 201, 461-477.

Lorez, M. (1995). Neural control of hindleg steering in flight in the locust. $J$. Exp. Biol. 198, 869-875.

Marden, J. H. (1987). Maximum lift production during takeoff in flying animals. J. Exp. Biol. 130, 235-258.

Maxworthy, T. (1979). Experiments on the Weis-Fogh mechanism of lift generation by insects in hovering flight. Part 1 . Dynamics of the 'fling'. $J$. Fluid Mech. 93, 47-63.

May, M. L. and Hoy, R. R. (1990). Leg-induced steering in flying crickets. J. Exp. Biol. 151, 485-488.

Robertson, R. and Johnson, A. (1993). Collision avoidance of flying locusts: steering torques and behaviour. J. Exp. Biol. 183, 35-60.

Ruppell, G. (1989). Kinematic analysis of symmetrical flight manoeuvres of Odonata. J. Exp. Biol. 144, 13-43.

Sedov, L. I. (1965). Two-Dimensional Problems in Hydrodynamics and Aerodynamics, pp. 20-30. New York: Interscience Publishers.

Spedding, G. R. (1993). On the significance of unsteady effects in the aerodynamic performance of flying animals. Contemp. Math. 141, 401-419.

Spedding, G. R. and Maxworthy, T. (1986). The generation of circulation and lift in a rigid two-dimensional fling. J. Fluid Mech. 165, 247-272.

Tu, M. S. and Dickinson, M. H. (1994). Modulation of negative work output from a steering muscle of the blowfly Calliphora vicina. J. Exp. Biol. 192, 207-224.

Vogel, S. (1967). Flight in Drosophila. III. Aerodynamic characteristics of fly wings and wing models. J. Exp. Biol. 46, 431-443.

Wakeling, J. E. and Ellington, C. P. (1997). Dragonfly flight. III. Lift and power requirements. J. Exp. Biol. 200, 583-600.

Wang, J. (2000). Vortex shedding and frequency selection in flapping flight. J. Fluid Mech. 410, 323-341.

Wilkin, P. J. (1990). The instantaneous force on a desert locust, Schistocerca gregaria (Orthoptera: Acrididae), flying in a wing tunnel. J. Kansas Ent. Soc. 63, 316-328.

Wortmann, M. and Zarnack, W. (1993). Wing movements and lift regulation in the flight of desert locusts. J. Exp. Biol. 182, 57-69.

Zanker, J. M. (1988). How does lateral abdomen deflection contribute to flight control of Drosophila melanogaster. J. Comp. Physiol. A 162 , 581-588.

Zanker, J. M. (1990a). The wing beat of Drosophila melanogaster. I. Kinematics. Phil. Trans. R. Soc. Lond. B 327, 1-18.

Zanker, J. M. (1990b). The wing beat of Drosophila melanogaster. III. Control. Phil. Trans. R. Soc. Lond. B 327, 45-64.

Zanker, J. M. and Götz, K. G. (1990). The wing beat of Drosophila melanogaster. II. Dynamics. Phil. Trans. R. Soc. Lond. B 327, 19-44. 